\title{
Towards adiabatic waveforms for inspiral into Kerr black holes: I. A new model of the source for the time-domain perturbation equation
}

\author{
Pranesh A. Sundararajan ${ }^{1}$, Gaurav Khanna ${ }^{2}$, and Scott A. Hughes ${ }^{1}$ \\ ${ }^{1}$ Department of Physics and MIT Kavli Institute, \\ MIT, 77 Massachusetts Ave., Cambridge, MA 02139 \\ ${ }^{2}$ Department of Physics, University of Massachusetts, Dartmouth, MA 02747
}

(Dated: September 18, 2018)

\begin{abstract}
We revisit the problem of the emission of gravitational waves from a test mass orbiting and thus perturbing a Kerr black hole. The source term of the Teukolsky perturbation equation contains a Dirac delta function which represents a point particle. We present a technique to effectively model the delta function and its derivatives using as few as four points on a numerical grid. The source term is then incorporated into a code that evolves the Teukolsky equation in the time domain as a $(2+1)$ dimensional PDE. The waveforms and energy fluxes are extracted far from the black hole. Our comparisons with earlier work show an order of magnitude gain in performance (speed) and numerical errors less than $1 \%$ for a large fraction of parameter space. As a first application of this code, we analyze the effect of finite extraction radius on the energy fluxes. This paper is the first in a series whose goal is to develop adiabatic waveforms describing the inspiral of a small compact body into a massive Kerr black hole.
\end{abstract}

PACS numbers: 04.25.Nx, 04.30.Db, 04.30.-w

\section{INTRODUCTION}

\section{A. Background}

The extreme mass ratio limit of binary systems - binaries with one mass far smaller than the other - has been a special focus of research in gravitation in recent years. This is in part because this problem is, at least formally, particularly clean and beautiful: the mass ratio allows us to treat the binary as an exact black hole solution plus a perturbation due to the secondary mass. Perturbative techniques can be used to analyze the system, making it (in principle at least) much more tractable than the general two-body problem in general relativity.

This limit is also of great astrophysical interest, as it perfectly describes capture binaries: binary systems created by the capture of stellar mass compact objects onto relativistic orbits of massive black holes in galaxy cores. Post formation, the evolution of such binaries is driven by gravitational-wave (GW) emission - the GW backreaction circularizes and shrinks the binaries, eventually driving the smaller body to plunge and merge with its larger companion. Such events are now believed to be relatively abundant (see Ref. [1] for up-to-date discussion and review of the relevant literature). Since the last year or so of the inspiral is likely to generate GWs that lie in the low-frequency band of space-based GW antennae such as LISA [2], extreme mass ratio inspirals (or EMRIs) are key targets for future GW observations.

This paper is the first in a series whose aim is to develop adiabatic EMRI waveforms. "Adiabatic" refers to the fact that they are computed using an approximation to the true equations of motion that takes advantage of the nearly periodic nature of the smaller body's motion on "short" timescales. This approximation fails to capture certain important aspects of the binary's evolution.
In particular, adiabatic waveforms only incorporate dissipative effects of the small body's perturbation - effects which cause radiation of energy and angular momentum to distant observers and down the hole, driving the orbit to decay. Conservative effects - effects which conserve energy and angular momentum, but push the orbit away from the geodesic trajectory of the background spacetime - are missed in this approach. It has been convincingly demonstrated [3] that conservative effects change orbital phasing in a way that could be observationally significant. The dissipative-only adiabatic approach to EMRI waveform generation is thus, by construction, somewhat deficient.

In our view, this deficiency is outweighed by the fact that it will produce waveforms that capture the spectral features of true waveforms - a complicated shape "colored" by the three fundamental orbital frequencies and their harmonics. Also, the adiabatic approach is likely to produce these waveforms on a relatively short timescale. Though not perfectly accurate, adiabatic waveforms will be an invaluable tool in the short term for workers developing a data analysis architecture for measuring EMRI events. In the long term, these waveforms may even be accurate enough to serve as "detection templates" for EMRI events. Measuring the characteristics of EMRI sources will require matching data with as accurate a model as can be made, and over as long a timespan as possible - perhaps a year or more. By contrast, detecting EMRI events does not require matching a signal with a template for such a long time [4]. For the short integration times needed for detection, work in progress indicates that conservative effects do not shift the phase so badly that the signal fails to match a template. What shift does accumulate due to conservative effects can be accomodated by systematic errors in source parameters, allowing detection to occur. (This is discussed in Ap- 
pendix A of Ref. [5], Chapter 4 of Ref. [6], and Refs. [7, 8], currently in preparation.)

\section{B. Our approach to adiabatic inspiral}

The approach which we advocate for building adiabatic waveforms uses a hybrid of frequency-domain and timedomain perturbation theory techniques. These two techniques have complementary strengths and weaknesses; by combining the best features of both toolsets, we hope to make waveforms that are as accurate as possible. Though a diversion from the main topic of this paper, this approach is a key motivation for our work. We thus ask the reader to indulge us as we briefly describe our rationale.

In the adiabatic limit and neglecting conservative effects, the separation of timescales means that orbits are, to high accuracy, simply geodesic trajectories of the spacetime on short timescales. The orbital decay that is driven by backreaction amounts to the system evolving from one geodesic orbit to another. Computing the effect of radiation reaction thus amounts to computing the sequence of orbits through which the system passes en route to the final plunge of the smaller body into the large black hole [9].

A geodesic orbit is characterized (up to initial conditions) by three constants: energy $E$; axial angular momentum $L_{z}$; and "Carter constant" $Q$ (see, e.g., 10], Chap. 33). Computing this sequence of orbits is equivalent to computing the rate at which these constants change due to radiative backreaction. In this picture, it is useful to regard each orbit $\left(E, L_{z}, Q\right)$ as a point in an orbital phase space, and to regard the rates at which they evolve, $\left(\dot{E}, \dot{L}_{z}, \dot{Q}\right)$, as defining a tangent vector to the trajectory an evolving system traces through this phase space. Adiabatic radiation reaction thus amounts to calculating this tangent at all orbits.

In the extreme mass ratio limit, the smaller body moves very slowly through orbit space - it spends many orbits in the vicinity of each $\left(E, L_{z}, Q\right)$. This slow evolution means that the tangent vector is most accurately represented by the average rate at which these constants evolve: $\left(\langle\dot{E}\rangle,\left\langle\dot{L}_{z}\right\rangle,\langle\dot{Q}\rangle\right)$, where the angle brackets denote an appropriate averaging with respect to the orbits. Such an averaging is defined in Ref. [5].

Once adiabatic radiation reaction data has been found for all orbits, it is straightforward to choose initial conditions and compute the worldline $\mathbf{z}(t)$ which an inspiralling body follows. In this framework, it is just a geodesic worldline with the constants slowly evolving:

$$
\mathbf{z}(t)=\mathbf{z}_{\text {geod }}\left[E(t), L_{z}(t), Q(t)\right] .
$$

This worldline can then be used to build the source term for the wave equation, allowing us to compute the gravitational waves generated as the small body spirals in. We note here that this approach is conceptually identical to the "kludge" presented in Ref. [11]. Indeed, the almost unreasonable success of kludge waveforms served as an inspiration for this formulation of inspiral ${ }^{1}$.

In the hybrid approach, a frequency-domain code would be used for the adiabatic radiation reaction, and a time-domain code used to generate the waves from a small body following the worldline that radiation reaction defines. Since any function built from bound Kerr black hole orbits has a spectrum that is fully described by three easily computed frequencies and their harmonics [12, 13], the averaging needed in this prescription is extremely fast and easy to compute in the frequency domain. Many harmonics may be needed, but each harmonic is independent of all others. Frequency-domain codes are thus easily parallelized and the calculation can be done very rapidly. In the time domain, averaging is much more cumbersome - a geodesic orbit and the radiation it generates must be followed over many orbits to insure that all beatings between different harmonics have been sampled. Convergence to the true average for quantities like $\langle\dot{E}\rangle$ will be slow for generic (inclined and eccentric) Kerr black hole orbits (the most interesting case, astrophysically).

By constrast, building the associated gravitational waveform with a frequency-domain code is rather cumbersome. One must build the Fourier expansion of the waves from many coefficients, and accurately sum them to produce the wave at any moment of time. The benefit of each harmonic being independent of all others is lost. In the time domain, building the waveform is automatic - modulo two time derivatives, the waveform is the observable that the code produces. Given a worldline, it is straightforward to build a source for the time-domain wave equation; one then cannot help but compute the waveform that source generates.

We are thus confident that by using both frequency and time-domain perturbation techniques, we can get the best of both worlds - letting each technique's complementary strengths shine to build EMRI waveforms that are as accurate as possible, in the context of the adiabatic approximation.

\section{This paper}

Key to the success of the hybrid approach is the development of fast, accurate codes for both frequency and time-domain approaches to black hole perturbation theory. First results from a frequency-domain code which can handle generic orbits have recently been presented [14], and the last major formal step (understanding the

\footnotetext{
1 The major difference between the hybrid inspiral described here and the kludge is that the hybrid inspiral aims to correctly solve a wave equation at all points along the orbit. The kludge instead uses a physically motivated approximate wave formula based on variation of the source's multipole moments, defined in a particular coordinate system.
} 
adiabatic evolution of Carter's constant $Q$ due to GW emission) is essentially in hand [5, 15, 16]. The frequencydomain side of this program is thus in a good state. Our goal now is to develop time-domain tools sufficiently robust and generic to handle the case of interest.

The major difficulty in building a time-domain perturbation code is the source term, representing the smaller member of the binary which perturbs the large black hole's spacetime. In the frequency domain, the small body is usually approximated as having zero spatial extent, and can be represented using delta functions (and their derivatives). One then constructs a Green's function from solutions of the source-free perturbation equation and integrates over the source. Thanks to the delta nature of the source in this representation, this integral can be done analytically. This trick cannot be done in the time domain - one must choose a functional form of the source which can be represented on a finite difference grid. The challenge is to pick a representation that accurately captures the very narrow spatial extent of the source, but is sufficiently smooth that the source does not seed excessive amounts of numerical error. This is particularly difficult for sources representing highly dynamic, generic Kerr black hole orbits in which the source rapidly moves across the grid.

Much recent success in this approach has come from representing the source as a truncated, narrow Gaussian [17]. Khanna [18] and Burko and Khanna [19] have so far examined some orbit classes (equatorial orbits, both circular and eccentric) and found that they can quickly and robustly generate waveforms from orbits around Kerr black holes. As a diagnostic of this technique, they compute the flux of energy carried by this radiation and find agreement with pre-existing frequency-domain calculations at the few percent level.

An interesting recent development is the use of finite element techniques to represent time-domain sources. Such methods are tailor made for resolving problems with multiple lengthscales, and as such may be ideal for the EMRI problem. Sopuerta and Laguna [20] have found that a finite element code makes it possible to represent the source with amazing accuracy - agreement with frequency-domain calculations at the few hundredths of a percent level seems common. To date, they have only examined binaries in which the larger black hole is nonrotating, but they argue convincingly [21] that the difficulties required to model Kerr perturbations should not be terribly difficult to surmount. These techniques are an extremely promising direction that is sure to develop extensively in the next several years.

Our goal in this paper is to develop another representation of the source term that is simpler (and concomitantly less accurate) than finite element methods, but that is developed somewhat more systematically than the truncated Gaussian. The key ingredient of this approach is an extension of a finite impulse representation of the Dirac delta function 23, 24]. In essence, one writes the discrete delta as a series of spikes on the finite differ- ence grid, with the largest spike centered at the argument of the delta, and with the spikes rapidly falling off away from this center. One chooses the magnitude of the spikes such that the delta function's integral properties are preserved, particularly the rule that

$$
\int d x f(x) \delta\left(x-x_{0}\right)=f\left(x_{0}\right) .
$$

The discrete delta described in Ref. 23] allows one to make a tradeoff between localization and smoothness one can smear the delta over $k$ points, choosing $k$ to be small if source sharpness is the key property needed, or allowing $k$ to expand if too much sharpness causes numerical problems. This representation introduces a kind of optimization parameter which one can engineer as needed to find the best compromise between smoothness and localization.

We extend the finite impulse representation of the delta described in 23, 24 in two important ways. First, the source term of the Teukolsky equation requires not just the delta, but also the delta's first and second derivatives. We therefore generalize this procedure to develop discrete delta derivatives. If the delta is represented by $k$ points, then both derivatives will require $k+2$ points. The guiding principle of this extension is again the notion that the integral properties of these functions must be preserved:

$$
\begin{gathered}
\int d x f(x) \delta^{\prime}\left(x-x_{0}\right)=-f^{\prime}\left(x_{0}\right), \\
\int d x f(x) \delta^{\prime \prime}\left(x-x_{0}\right)=f^{\prime \prime}\left(x_{0}\right) .
\end{gathered}
$$

(Here, prime means $d / d x$.)

If the discrete delta function does not lie precisely on a grid point, then one must use interpolation to appropriately weight impulse functions from the neighboring grid points. Our second extension of Ref. [23] is to introduce higher order interpolation (cubic) which offers another way to trade smoothness for localization. This is particularly valuable when (as in our application) the source is coupled to a wave equation.

We test this representation by developing a new timedomain Teukolsky equation solver which uses this form of the delta for its source (the " $\delta$-code") and comparing to a well-established code (see, e.g., [17, 18, 19]) which uses a truncated Gaussian (the "G-code"). The G-code has been described in detail in a previous publication [17]; for the purpose of this paper, the most salient feature of this code is how it represents the source term. The G-code begins with the following approximation to the Dirac delta function:

$$
\delta[x-x(t)] \simeq \frac{1}{\sqrt{2 \pi} \sigma} \exp \left(-\frac{[x-x(t)]^{2}}{2 \sigma^{2}}\right) .
$$

[Cf. Ref. [17], Eq. (19).] The width $\sigma$ is chosen to be small enough that this delta only spreads across a few 
grid zones. The Teukolsky equation source is then built from this Gaussian representation and its derivatives.

The $\delta$-code by contrast uses the representation described in detail in the following sections of this paper a representation that is discrete by design, rather than a discretization of a continuous delta approximation. The principle advantage of this form seems to be that it makes it possible to rigorously enforce integral identities involving the delta plus its derivatives.

There are a few other minor differences between these two codes, which are artifacts of the codes' independent developments. Chief among these differences are the use of slightly different axial coordinates (the G-code uses the usual Boyer-Lindquist coordinate $\phi$; the $\delta$-code follows Ref. 25] and uses a coordinate $\tilde{\phi}$ defined in Sec. II A), and the use of slightly different fundamental "fields" (i.e., slightly different representations of the Weyl curvature scalar $\psi_{4}$ which the Teukolsky equation governs). There are also some differences in the way the two codes implement boundary conditions. We present a detailed comparison of the results from the two codes in Sec. IV. It's worth pointing out that that we also have taken the Gcode and replaced its source term with that used by the $\delta$-code. This exercise confirmed all of the results we obtained with the $\delta$-code, demonstrating that these minor differences had no impact on our results.

As a proof-of-principle check of this idea's validity, we restrict our present analysis to circular, equatorial orbits. The results from both codes are then compared against frequency-domain results. Flux of energy carried by gravitational waves is a very useful benchmark with which to diagnose a perturbation theory code's accuracy (especially for very simple orbits when averaging is easy both in time and frequency domains). In all cases, we find (after some experimentation to optimize our discrete delta) that this new source form is more accurate (typically by factors of $2-5$ ) and faster (often by factors of about 10) than the truncated Gaussian. For our purpose, it appears that this form of source function will be very well-suited to serve as the core of the time-domain portion of our hybrid approach to EMRI waveforms.

Future papers in this series will then apply this technique to flesh out the hybrid approach. Our first followup will examine how well this source works for highly dynamical trajectories - generic (inclined and eccentric) geodesic orbits, plunging orbits, and non-geodesic trajectories (standing in for orbits that evolve due to radiation reaction). Early results from this analysis indicate that the discrete delta source term handles such orbits very robustly, validating earlier results for eccentric equatorial orbits [19]; work is in progress to extend this to generic orbits. We will then begin developing hybrid EMRI waveforms in earnest, using frequency-domain tools to compute the effects of radiation reaction, building an inspiral worldline from those effects, and finally computing the waveform with our time-domain code.

\section{Organization of this paper}

The remainder of this paper is organized as follows. Section III reviews how one solves the Teukolsky equation in the time domain, introducing the equation itself, specializing to the form that we use for our calculations, and showing how to extract waveforms and fluxes from its solutions. We first review in Sec. II A how one solves for the homogeneous (source-free) form of the Teukolsky equation, an important first step to developing a robust solver for the sourced case. We follow very closely the procedure laid out in Ref. 25]; this section is thus largely a review and summary of that paper (with a few minor corrections noted). Section II B then describes in detail the form of the source term that applies when perturbations arise from an orbiting body.

The need to model this source using a delta function motivates Sec. III, our model for a discrete delta and its derivatives. This section presents the key new idea of this paper. After describing the basic idea behind our discrete delta, we first present in some detail (Sec. III A) an extremely simple two-point discrete delta function. This illustrates the concepts and principles of this approach. We then generalize this idea to a multiple point delta in Sec. IIIB, and then show how to smooth things with higher order interpolation in Sec. IIIC. Some preliminary issues related to the convergence of quantities computed using the discrete delta are introduced in Sec. IIID.

We test this delta representation in Sec. IV. examining how the various methods we develop work at describing the Teukolsky source function. Section IV A first compares the different discrete delta functions with each other, demonstrating how the different approaches change the quality and accuracy of our results. Based on this analysis, we choose to use the high order (cubic) delta described in Sec. IIC in the remainder of our work. We then examine the convergence of our code, demonstrating second-order convergence in Sec. IVB. Finally, in Sec. IV C we compare the discrete delta with the Gaussian source function, demonstrating explicitly how this new representation improves both the code's speed and accuracy.

Our benchmark for evaluating our results is to compare the energy flux carried by the system's emitted gravitational waves to results obtained using a frequencydomain code [14, 27]. This operation requires us to extract these waves at a particular finite radius. Section $\nabla$ examines the dependence of these fluxes as a function of extraction radius, and finds that they are very well fit by a simple power law. Using this law, we can easily extrapolate our results to very large radius; doing so greatly improves agreement with frequency-domain results, typically indicating that our errors are significantly smaller than $1 \%$ for a large fraction of parameter space.

Concluding discussion in given in Sec. VI. Besides summarizing the major findings of this analysis, we discuss in some detail future projects to which we intend to 
apply this new computational technology.

\section{NUMERICAL IMPLEMENTATION OF THE TEUKOLSKY EQUATION IN THE TIME DOMAIN}

Here we describe the evolution algorithm used in the $\delta$-Code, built using a two step Lax-Wendroff algorithm. Our notation and approach closely follow that used in [25]; some of this section therefore can be considered a summary of that paper. All details related to the G-Code were described in [17].

Teukolsky derived a master equation that describes perturbations due to scalar, vector and tensor fields in the vicinity of Kerr black holes in [22, 29]. In BoyerLindquist coordinates, this equation reads

$$
\begin{aligned}
& -\left[\frac{\left(r^{2}+a^{2}\right)^{2}}{\Delta}-a^{2} \sin ^{2} \theta\right] \partial_{t t} \Psi-\frac{4 M a r}{\Delta} \partial_{t \phi} \Psi \\
& -2 s\left[r-\frac{M\left(r^{2}-a^{2}\right)}{\Delta}+i a \cos \theta\right] \partial_{t} \Psi \\
& +\Delta^{-s} \partial_{r}\left(\Delta^{s+1} \partial_{r} \Psi\right)+\frac{1}{\sin \theta} \partial_{\theta}\left(\sin \theta \partial_{\theta} \Psi\right)+ \\
& {\left[\frac{1}{\sin ^{2} \theta}-\frac{a^{2}}{\Delta}\right] \partial_{\phi \phi} \Psi} \\
& +2 s\left[\frac{a(r-M)}{\Delta}+\frac{i \cos \theta}{\sin ^{2} \theta}\right] \partial_{\phi} \Psi \\
& -\left(s^{2} \cot ^{2} \theta-s\right) \Psi=-4 \pi\left(r^{2}+a^{2} \cos ^{2} \theta\right) T,
\end{aligned}
$$

where $M$ is the mass of the black hole, $a$ its angular momentum per unit mass, $\Delta=r^{2}-2 M r+a^{2}=$ $\left(r-r_{+}\right)\left(r-r_{-}\right), r_{ \pm}=M \pm \sqrt{M^{2}-a^{2}}$ and $s$ is the "spin weight" of the field. The $s= \pm 2$ versions of these equations describe perturbations to the Weyl curvature tensor, in particular the radiative degrees of freedom $\psi_{0}$ and $\psi_{4}$. That is, $\Psi=\psi_{0}$ for $s=+2$, and $\Psi=\rho^{-4} \psi_{4}$ for $s=-2$, with $\rho=-1 /(r-i a \cos \theta)$. The $T$ in the RHS of this equation depends on the details of the perturbing source. It is here that the Dirac delta function and its derivatives enter. A discussion of $T$ is postponed to the latter half of this section, after we discuss the numerical evolution of the homogeneous Teukolsky equation.

Gravitational waves, $h_{+}$and $h_{\times}$as well as the energy flux $d E / d t$ [32, 33], can be obtained far away from the system by using $s=-2$ in Eq. (2.1) and then identifying

$$
\begin{gathered}
\psi_{4}=\frac{1}{2}\left(\frac{\partial^{2} h_{+}}{\partial t^{2}}-i \frac{\partial^{2} h_{\times}}{\partial t^{2}}\right) \\
\frac{d E}{d t}=\lim _{r \rightarrow \infty}\left[\frac{1}{4 \pi r^{6}} \int_{\theta} \int_{\phi} \sin \theta d \theta d \phi\left|\int_{-\infty}^{t} d \tilde{t} \Psi(\tilde{t}, r, \theta, \phi)\right|^{2}\right] \\
=\lim _{r \rightarrow \infty}\left[\frac{1}{2} \int_{\theta} \sin \theta d \theta\left|\int_{-\infty}^{t} d \tilde{t} \Phi(\tilde{t}, r, \theta)\right|^{2}\right]
\end{gathered}
$$

The $\theta$ and $\phi$ directions are taken with respect to the black hole's spin axis; the function $\Phi(t, r, \theta)$ is a reweighting of the field $\Psi$ which we define precisely in Eq. (2.8) below.

\section{A. Homogeneous Teukolsky equation}

Reference 25] demonstrated stable numerical evolution of (2.1) for $s=-2$. The $\delta$-code has been built using the algorithm presented in [25], after accounting for some typographical errors, which are also discussed in [30]. The contents of this section are largely review of the results presented in [25]; as such, our discussion is particularly brief here.

Our code uses the tortoise coordinate $r^{*}$ in the radial direction, and azimuthal coordinate $\tilde{\phi}$; these coordinates are related to the usual Boyer Lindquist quantities by

$$
\begin{aligned}
d r^{*}= & \frac{r^{2}+a^{2}}{\Delta} d r \\
\Rightarrow r^{*}= & r+\frac{2 M r_{+}}{r_{+}-r_{-}} \ln \frac{r-r_{+}}{2 M} \\
& -\frac{2 M r_{-}}{r_{+}-r_{-}} \ln \frac{r-r_{-}}{2 M}
\end{aligned}
$$

and

$$
\begin{aligned}
d \tilde{\phi} & =d \phi+\frac{a}{\Delta} d r \\
\Rightarrow \tilde{\phi} & =\phi+\frac{a}{r_{+}-r_{-}} \ln \frac{r-r_{+}}{r-r_{-}} .
\end{aligned}
$$

Following [25], we factor out the azimuthal dependence and use the ansatz,

$$
\Psi\left(t, r^{*}, \theta, \tilde{\phi}\right)=e^{i m \tilde{\phi}} r^{3} \Phi\left(t, r^{*}, \theta\right) .
$$

Defining

$$
\begin{aligned}
\Pi & \equiv \partial_{t} \Phi+b \partial_{r^{*}} \Phi, \\
b & \equiv \frac{r^{2}+a^{2}}{\Sigma},
\end{aligned}
$$

and

$$
\Sigma^{2} \equiv\left(r^{2}+a^{2}\right)^{2}-a^{2} \Delta \sin ^{2} \theta
$$

allows the Teukolsky equation to be rewritten as

$$
\partial_{t} \boldsymbol{u}+\boldsymbol{M} \partial_{r *} \boldsymbol{u}+\boldsymbol{L} \boldsymbol{u}+\boldsymbol{A} \boldsymbol{u}=\boldsymbol{T},
$$

where

$$
\boldsymbol{u} \equiv\left\{\Phi_{R}, \Phi_{I}, \Pi_{R}, \Pi_{I}\right\}
$$

is the solution vector. The subscripts $R$ and $I$ refer to the real and imaginary parts respectively. (Note that the Teukolsky function $\Psi$ is a complex quantity.) The matrices $\boldsymbol{M}, \boldsymbol{A}$ and $\boldsymbol{L}$ are

$$
\boldsymbol{M} \equiv\left(\begin{array}{cccc}
b & 0 & 0 & 0 \\
0 & b & 0 & 0 \\
m_{31} & m_{32} & -b & 0 \\
-m_{32} & m_{31} & 0 & -b
\end{array}\right)
$$




$$
\boldsymbol{A} \equiv\left(\begin{array}{cccc}
0 & 0 & -1 & 0 \\
0 & 0 & 0 & -1 \\
a_{31} & a_{32} & a_{33} & a_{34} \\
-a_{32} & a_{31} & -a_{34} & a_{33}
\end{array}\right)
$$

and

$$
\boldsymbol{L} \equiv\left(\begin{array}{cccc}
0 & 0 & 0 & 0 \\
0 & 0 & 0 & 0 \\
l_{31} & 0 & 0 & 0 \\
0 & l_{31} & 0 & 0
\end{array}\right)
$$

where

$$
\begin{aligned}
m_{31}= & -b c_{1}+b \partial_{r^{*}} b+c_{2} \\
m_{32}= & b c_{3}+2 a m\left(r^{2}+a^{2}\right) / \Sigma^{2} \\
a_{31}= & \Delta \frac{m^{2}+2 \cos \theta s m+\cos ^{2} \theta s^{2}-\sin ^{2} \theta s}{\Sigma^{2} \sin ^{2} \theta} \\
& -6 \Delta \frac{a^{2}+r(r(s+2)-M(s+3))}{r^{2} \Sigma^{2}} \\
a_{32}= & \frac{4 M(r-1) \operatorname{sma} M+(6 a m \Delta) / r}{\Sigma^{2}}, \\
a_{33}= & c_{1}, \\
a_{34}= & -c_{3}, \\
l_{31}= & -\frac{\Delta}{\Sigma^{2}} \frac{\partial^{2}}{\partial \theta^{2}}-\cot \theta \frac{\Delta}{\Sigma^{2}} \frac{\partial}{\partial \theta}, \\
c_{1}= & 2 s\left(-3 M r^{2}+M a^{2}+r^{3}+r a^{2}\right) / \Sigma^{2} \\
c_{2}= & -2 \frac{r \Delta(1+s)-\left(a^{2}-r^{2}\right) M s}{\Sigma^{2}}-\frac{6 \Delta b}{r \Sigma} \\
c_{3}= & 2 a(2 r M m+\Delta s \cos \theta) / \Sigma^{2} .
\end{aligned}
$$

The equations above have been written such that the typographical errors in [25]'s $a_{31}, a_{32}, a_{34}$ and $c_{2}$ are obvious. It turns out that the coefficients listed in 25] are correct when the ansatz $\Psi\left(t, r^{*}, \theta, \tilde{\phi}\right)=e^{i m \tilde{\phi}} \Phi\left(t, r^{*}, \theta\right)$ is used. $\boldsymbol{T}$ is a quantity contructed from the source term and is discussed in the latter half of this section.

Rewriting Eq. (2.12) as

$$
\partial_{t} \boldsymbol{u}+\boldsymbol{D} \partial_{r^{*}} \boldsymbol{u}=\boldsymbol{S},
$$

where

$$
\begin{gathered}
\boldsymbol{D} \equiv\left(\begin{array}{cccc}
b & 0 & 0 & 0 \\
0 & b & 0 & 0 \\
0 & 0 & -b & 0 \\
0 & 0 & 0 & -b
\end{array}\right), \\
\boldsymbol{S}=\boldsymbol{T}-(\boldsymbol{M}-\boldsymbol{D}) \partial_{r^{*}} \boldsymbol{u}-\boldsymbol{L} \boldsymbol{u}-\boldsymbol{A u},
\end{gathered}
$$

and subjecting it to Lax-Wendroff iterations produces stable time-evolutions. Each Lax-Wendroff iteration consists of two steps. In the first step, the solution vector between grid points is obtained from

$$
\begin{aligned}
\boldsymbol{u}_{i+1 / 2}^{n+1 / 2}= & \frac{1}{2}\left(\boldsymbol{u}_{i+1}^{n}+\boldsymbol{u}_{i}^{n}\right)- \\
& \frac{\delta t}{2}\left[\frac{1}{\delta r^{*}} \boldsymbol{D}_{i+1 / 2}^{n}\left(\boldsymbol{u}_{i+1}^{n}-\boldsymbol{u}_{i}^{n}\right)-\boldsymbol{S}_{i+1 / 2}^{n}\right] .
\end{aligned}
$$

This is used to compute the solution vector at the next time step,

$\boldsymbol{u}_{i}^{n+1}=\boldsymbol{u}_{i}^{n}-\delta t\left[\frac{1}{\delta r^{*}} \boldsymbol{D}_{i}^{n+1 / 2}\left(\boldsymbol{u}_{i+1 / 2}^{n+1 / 2}-\boldsymbol{u}_{i-1 / 2}^{n+1 / 2}\right)-\boldsymbol{S}_{i}^{n+1 / 2}\right]$.

The angular subscripts are dropped here for clarity. All angular derivatives were computed using second order centered finite difference expressions. Notice that the matrices $\boldsymbol{D}, \boldsymbol{A}$ and $\boldsymbol{M}$ are time independent. In addition, the time stepping must satisfy the Courant-FriedrichsLewy condition [25], $\delta t \leq \max \left\{\delta r^{*}, 5 M \delta \theta\right\}$, where $\delta t$ is the time step ${ }^{2}$.

Following [25], we set $\Phi$ and $\Pi$ to zero on the inner and outer radial boundaries. While the aymptotic behavior

$$
\lim _{r^{*} \rightarrow-\infty}|\Psi| \propto \Delta^{-s}
$$

makes this condition reasonably accurate at the inner boundary, it is clearly unphysical at the outer boundary. By placing our outer boundary sufficiently far, error due to our outer boundary condition can be made unimportant; reflections from the outer boundary have no important impact on our results. Symmetry of the spheroidal harmonics is used to determine the angular boundary conditions. For even $|m|$ modes, we have $\partial_{\theta} \Phi=0$ at $\theta=0, \pi$. On the other hand, $\Phi=0$ at $\theta=0, \pi$ for modes of odd $|m|$.

As a test of our evolution equation, we have examined source-free field evolution (setting $\boldsymbol{T}=0$ ) for a variety of initial data, in particular comparing extensively with the results of [25]. As an example of one of our tests, we take initial data corresponding to an ingoing, narrow Gaussian pulse. This data perturbs the black hole, causing it to ring down according to its characteristic quasi-normal frequencies. We find extremely good agreement in mode amplitude and evolution (typically $\sim 1 \%$ error) with results from [25]. Figure 1 shows the result of such a test, illustrating the quasi-normal ringing and power law tail for the $l=2, m=0$ mode of a black hole with spin parameter $a=0.9$.

\section{B. The source term}

We now consider the source term, $T$, of Eq. (2.1). It is given by

$$
\begin{aligned}
T= & 2 \rho^{-4} T_{4}, \\
T_{4}= & (\tilde{\Delta}+3 \gamma-\bar{\gamma}+4 \mu+\bar{\mu})(\tilde{\Delta}+2 \gamma-2 \bar{\gamma}+\bar{\mu}) T_{\bar{m} \bar{m}} \\
& -(\tilde{\Delta}+3 \gamma-\bar{\gamma}+4 \mu+\bar{\mu})(\bar{\delta}-2 \bar{\tau}+2 \alpha) T_{n \bar{m}} \\
& +(\bar{\delta}-\bar{\tau}+\bar{\beta}+3 \alpha+4 \pi)(\bar{\delta}-\bar{\tau}+2 \bar{\beta}+2 \alpha) T_{n n} \\
& -(\bar{\delta}-\bar{\tau}+\bar{\beta}+4 \pi)(\tilde{\Delta}+2 \gamma+2 \bar{\mu}) T_{n \bar{m}} .
\end{aligned}
$$

\footnotetext{
2 Conducting a von Neumann local stability analysis on all the points of our numerical grid yields that this condition is sufficient for stable evolutions. See reference [25] for more details.
} 

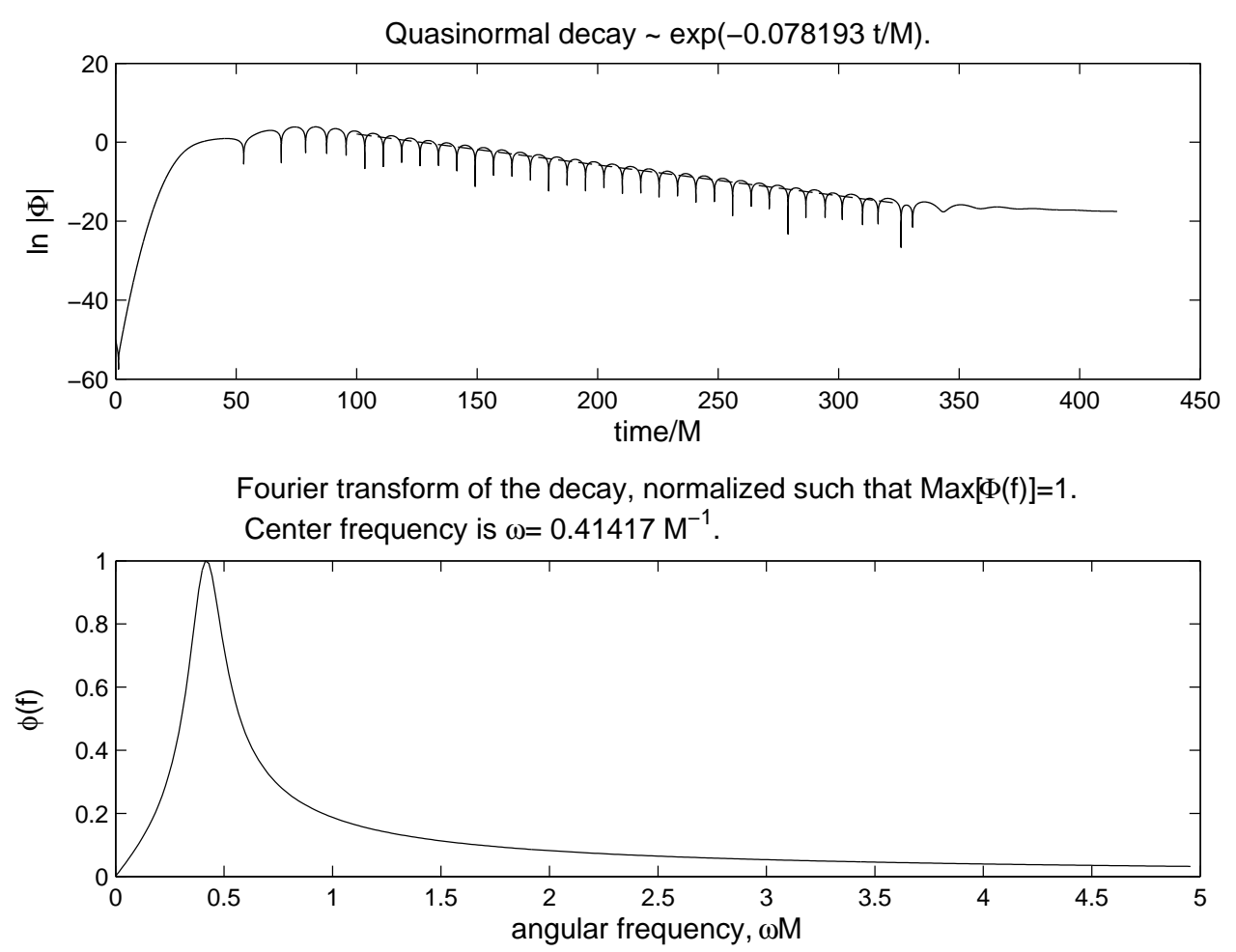

FIG. 1: Illustrations of quasi-normal ringing for a black hole with $a / M=0.9$; the $l=2, m=0$ mode is shown here. Top panel: Evolution of the magnitude of the Teukolsky function, extracted at $r=20 M, \theta=\pi / 2$. We plot the time evolution of $\ln |\Phi|$ at this position. Overplotted on this curve (dashed line) is a function $\propto \exp (-0.078193 t / M)$, demonstrating that we recover the expected decay law with a damping time $\tau=12.789 M$. Bottom panel: Magnitude of the Fourier transform of $\Phi(t)$. Notice that it peaks at $\omega=0.41417 / M$. These results for $\omega$ and $\tau$ are in excellent agreement with the expected values of $(\omega M, M / \tau)=(0.41,-0.078)$ from Ref. [26] for quasi-normal ringing of the $l=2, m=0$ mode for $a=0.9$.

Reference [22] provides definitions for the various quantities which appear in Eqs. (2.34) and (2.34). Of particular importance are the quantities $T_{n n}, T_{n \bar{m}}$, and $T_{\bar{m} \bar{m}}$, given by contracting the stress energy tensor for the orbiting body with the Newman-Penrose null-tetrad legs $n^{\mu}$ and $\bar{m}^{\mu}$ :

$$
\begin{aligned}
n^{\mu} \doteq & \left(\frac{\rho^{2}\left(r^{2}+a^{2}\right)}{2},-\frac{\Delta \rho^{2}}{2}, 0, \frac{a \rho^{2}}{2}\right), \\
\bar{m}^{\mu} \doteq & \frac{1}{\sqrt{2}(r-i a \cos \theta)} \\
& (-i a \sin \theta, 0,1,-i \csc \theta),
\end{aligned}
$$

where $\Delta=r^{2}-2 M r+a^{2}$ and $\rho^{-2}=r^{2}+a^{2} \cos ^{2} \theta$.

Also of great importance here are the Newman-Penrose operators $\tilde{\Delta}$ and $\bar{\delta}$ :

$$
\begin{aligned}
\tilde{\Delta}= & n^{\mu} \frac{d}{d x^{\mu}} \\
= & \frac{\rho^{2}\left(r^{2}+a^{2}\right)}{2} \frac{d}{d t}-\frac{\rho^{2} \Delta}{2} \frac{d}{d r}+\frac{a i m \rho^{2}}{2} \\
\equiv & \Delta_{t}+\Delta_{r}+\Delta_{\phi} ; \\
\bar{\delta}= & \bar{m}^{\mu} \frac{d}{d x^{\mu}} \\
= & -\frac{i a \sin \theta \rho^{2}(r+i a \cos \theta)}{\sqrt{2}} \frac{d}{d t} \\
& +\frac{(r+i a \cos \theta) \rho^{2}}{\sqrt{2}} \frac{d}{d \theta}+\frac{m \rho^{2}(r+i a \cos \theta)}{\sqrt{2} \sin \theta} \\
\equiv & \bar{\delta}_{t}+\bar{\delta}_{\theta}+\bar{\delta}_{\phi} .
\end{aligned}
$$

(The operator $\tilde{\Delta}$ is normally written without the tilde; we have added it here to avoid confusion with $\Delta=r^{2}-$ $2 M r+a^{2}$.)

To proceed, we next must analyze the stress energy tensor describing the small body. A point body of mass $\mu$ disturbing the Kerr spacetime is given by

$$
T_{\mu \nu}=\frac{\mu u_{\mu} u_{\nu} \rho^{2}}{u^{t} \sin \theta} \delta[r-R(t)] \delta[\theta-\Theta(t)] \delta[\phi-\Phi(t)],
$$


where $u^{\mu}=d x^{\mu} / d \tau$, and where $[R(t), \Theta(t), \Phi(t)]$ describe the Boyer-Lindquist coordinate worldline of the small body. Due to axial symmetry of the Kerr spacetime, the only $\phi$ dependence in the stress-energy tensor comes from $\delta[\phi-\Phi(t)]$. Writing

$$
T_{\mu \nu}=\sum_{m} T_{\mu \nu}^{m} e^{i m \phi}
$$

and using the fact that

$$
\delta[\phi-\Phi(t)]=\frac{1}{2 \pi} \sum_{m} e^{i m[\phi-\Phi(t)]},
$$

we find

$$
T_{\mu \nu}^{m}=\frac{\mu u_{\mu} u_{\nu} \rho^{2}}{u^{t} \sin \theta} \delta[r-R(t)] \delta[\theta-\Theta(t)] e^{-i m \Phi(t)} .
$$

Using this expansion for $T_{\mu \nu}$, it is a simple matter to construct $T_{n n}=n^{\mu} n^{\nu} T_{\mu \nu}, T_{n \bar{m}}=n^{\mu} \bar{m}^{\nu} T_{\mu \nu}$, and $T_{\bar{m} \bar{m}}=\bar{m}^{\mu} \bar{m}^{\nu} T_{\mu \nu}$. We then insert these terms into Eqs. (2.34). Using the chain rule repeatedly leaves us with a (rather complicated) expression involving radial and $\theta$ derivatives of Dirac delta functions. We thus face the task of representing the delta function and its derivatives accurately on a numerical grid. This is the major innovation of this paper, and is discussed in detail in the following section.

It should be noted at this point that, since the Teukolsky equation is most naturally written in terms of the tortoise coordinate $r^{*}$, we must describe the radial behavior of the source term in $r^{*}$ as well. To this end, we replace the radial delta function and all radial derivatives as follows:

$$
\begin{aligned}
\delta[r-R(t)] & =\frac{\delta\left[r^{*}-R^{*}(t)\right]}{\left|d r / d r^{*}\right|} \\
\frac{d}{d r} & =\frac{r^{2}+a^{2}}{\Delta} \frac{d}{d r^{*}}
\end{aligned}
$$

Finally, in our numerical implementation, we define the vector $\boldsymbol{T}$ appearing in Eq. (2.7) as

$$
\boldsymbol{T} \doteq[0,0, \operatorname{Re}(\hat{T}), \operatorname{Im}(\hat{T})]
$$

where

$$
\begin{aligned}
\hat{T}= & \frac{4 \pi \Delta\left(r^{2}+a^{2} \cos ^{2} \theta\right)}{r^{3}\left[\left(r^{2}+a^{2}\right)^{2}-a^{2} \Delta \sin ^{2} \theta\right]} \times \\
& \exp \left(-i m \frac{a}{r_{+}-r_{-}} \ln \left[\frac{r-r_{+}}{r-r_{-}}\right]\right)\left[2 \rho^{-4} T_{4}\right] .
\end{aligned}
$$

The exponential factor in this expression corrects for the fact that the evolution code uses the azimuthal variable $\tilde{\phi}$, but the source term is expanded in $\phi$.

\section{THE DISCRETE DELTA FUNCTION AND ITS DERIVITATIVES}

As pointed out in the previous section, the Dirac delta function enters the Teukolsky equation because we approximate the perturbing mass by a point particle. By its definition as an integrable singularity, the delta function is very difficult to represent on a finite difference grid. The best we can hope to do is to develop a model function that captures its most important features, particularly localization to a very small spatial region, as well as integrability and derivative properties. The following three subsections describe the model for the delta function we have developed. We first describe a very basic model that demonstrates how to satisfy our criteria in Sec. III A. In Sec. II B and IIIC, we then refine this basic model. These refinements have been found to improve the overall accuracy of the code.

The discrete delta function approach we use is inspired by the work presented in Ref. [23]. Our technique can be considered an extension of that used in 23]; in particular, they do not develop delta function derivatives, nor do they implement all the refinements discussed in $\amalg$ and III C. Nonetheless, Ref. 23] played an extremely important role in developing the foundations of our work.

Before turning to a detailed discussion of our techniques for modeling the delta function on a numerical grid, we first mention some general considerations pertaining to delta functions on a finite difference grid. For concreteness, consider a function and delta combination, $f(x) \delta(x-\alpha)$. The function $f(x)$ is taken to be known, and can be calculated for any $x$. For the sake of argument, let the delta be modelled by two coefficients $\delta_{k}$ and $\delta_{k+1}$ on grid points $x_{k}$ and $x_{k+1}$ respectively; the delta is taken to be zero everywhere else. (This in fact pertains to the form of the delta discussed in Sec. III A.)

Now imagine integrating $f(x) \delta(x-\alpha)$ over all $x$. Analytically, we know that this should give us $f(\alpha)$. Numerically integrating this on our grid gives us

$$
\begin{aligned}
h \sum_{i} f\left(x_{i}\right) \delta\left(x_{i}-\alpha\right)= & h\left[f\left(x_{k}\right) \delta_{k}\right. \\
& \left.+f\left(x_{k+1}\right) \delta_{k+1}\right] .
\end{aligned}
$$

This equation suggests that the numerical integral approximates $f(\alpha)$ by interpolating between grid points $x_{k}$ and $x_{k+1}$. If $f(x)$ is rapidly varying, this interpolation may not be accurate enough; this is sure to be a source of error as we integrate our PDE forward in time.

Great improvement can be achieved by enforcing the well-known identity

$$
f(x) \delta(x-\alpha)=f(\alpha) \delta(x-\alpha) .
$$


The numerical integral now becomes

$$
\begin{aligned}
h \sum_{i} f(\alpha) \delta\left(x_{i}-\alpha\right) & =h\left[f(\alpha) \delta_{k}+f(\alpha) \delta_{k+1}\right] \\
& =f(\alpha) h\left(\delta_{k}+\delta_{k+1}\right) \\
& =f(\alpha),
\end{aligned}
$$

and the identity is preserved exactly. In the last step, we use the discrete analog of the property

$$
\int d x \delta(x-\alpha)=1
$$

Similar identities can be used on the delta function derivatives:

$$
\begin{aligned}
f(x) \delta^{\prime}(x-\alpha)= & f(\alpha) \delta^{\prime}(x-\alpha) \\
& -f^{\prime}(\alpha) \delta(x-\alpha) \\
f(x) \delta^{\prime \prime}(x-\alpha)= & f(\alpha) \delta^{\prime \prime}(x-\alpha)-2 f^{\prime}(\alpha) \delta^{\prime}(x-\alpha) \\
& +f^{\prime \prime}(\alpha) \delta(x-\alpha) .
\end{aligned}
$$

We recommend using these identities as much as possible when numerically implementing the algorithms sketched in the following three subsections.

\section{A. A simple numerical delta function}

Consider the function $\delta(x-\alpha)$, where $x_{k} \leq \alpha \leq x_{k+1}$; i.e, $\alpha$ lies between two discrete grid points. Let $h=$ $x_{k+1}-x_{k}=x_{k}-x_{k-1}$ be the grid resolution. We use the following integral to define the delta function:

$$
\int_{\alpha-\epsilon}^{\alpha+\epsilon} d x f(x) \delta(x-\alpha)=f(\alpha)
$$

where $\epsilon>0$ and $f(x)$ is any well behaved function. This means that $\delta(x-\alpha)$ is zero everywhere, except at $x=$ $\alpha$, where it is singular. Translating this integral to a summation, we have:

$$
\begin{aligned}
\int_{\alpha-\epsilon}^{\alpha+\epsilon} d x f(x) \delta(x-\alpha) & \simeq h \sum_{i} f\left(x_{i}\right) \delta_{i} \\
\Rightarrow f(\alpha) & \simeq h \sum_{i} f\left(x_{i}\right) \delta_{i},
\end{aligned}
$$

where $\delta_{i}$ is the discrete delta defined on the grid. Since $\alpha$ does not necessarily lie on a gridpoint, we can linearly interpolate to find:

$$
\begin{aligned}
f(\alpha)= & \frac{f\left(x_{k+1}\right)-f\left(x_{k}\right)}{h}\left(\alpha-x_{k}\right)+f\left(x_{k}\right) \\
& +O\left(h^{2}\right) .
\end{aligned}
$$

Substituting this back into our earlier expression and comparing coefficients, we have

$$
\begin{aligned}
\delta_{i} & =\frac{\alpha-x_{k}}{h^{2}} \text { for } i=k+1 \\
& =\frac{x_{k+1}-\alpha}{h^{2}} \text { for } i=k \\
& =0 \text { everywhere else } .
\end{aligned}
$$

Notice that if $\alpha=x_{k}$, then $\delta_{i}=1 / h$ for $i=k$, but is zero everywhere else; a similar result holds if $\alpha=x_{k+1}$. This reproduces our intuitive notion that the delta function is zero everywhere except at a single point, and that it integrates to unity. We take the viewpoint that the integrability of the delta function is its key defining property, using this rule to derive the results presented below. This is the approach that was used in Ref. [23].

Another approach to defining a numerical delta function, suggested in [18], is to first define a step function on the grid, and then use finite differencing to obtain the delta and its derivatives. The approach described above matches this proposal when $\alpha$ lies exactly on a grid point.

We can proceed in a similar fashion to find formulae for the derivatives. Let us define

$$
\gamma=\frac{x_{k+1}-\alpha}{h}=1-\frac{\alpha-x_{k}}{h} .
$$

Again, we start from the defining integrals,

$$
\begin{aligned}
& \int d x f(x) \delta^{\prime}(x-\alpha)=-f^{\prime}(\alpha), \\
& \int d x f(x) \delta^{\prime \prime}(x-\alpha)=f^{\prime \prime}(\alpha) .
\end{aligned}
$$

Note that a prime denotes $d / d x$. Our goal is to derive a form which enforces these integrals in summation form:

$$
\begin{aligned}
h \sum_{i} f\left(x_{i}\right) \delta_{i}^{\prime} \simeq & -f^{\prime}(\alpha) \\
= & -h \sum_{i} f^{\prime}\left(x_{i}\right) \delta_{i} \\
= & -\gamma f^{\prime}\left(x_{k}\right) \\
& -(1-\gamma) f^{\prime}\left(x_{k+1}\right)+O\left(h^{2}\right) ;
\end{aligned}
$$

$$
\begin{aligned}
h \sum_{i} f\left(x_{i}\right) \delta_{i}^{\prime \prime} \simeq & f^{\prime \prime}(\alpha) \\
= & h \sum_{i} f^{\prime \prime}\left(x_{i}\right) \delta_{i} \\
= & \gamma f^{\prime \prime}\left(x_{k}\right)+ \\
& (1-\gamma) f^{\prime \prime}\left(x_{k+1}\right)+O\left(h^{2}\right)
\end{aligned}
$$

The derivatives of $f\left(x_{k}\right)$ are given by the finite difference formulae,

$$
\begin{aligned}
f^{\prime}\left(x_{k}\right)= & \frac{f\left(x_{k+1}\right)-f\left(x_{k-1}\right)}{2 h}+O\left(h^{2}\right), \\
f^{\prime \prime}\left(x_{k}\right)= & \frac{f\left(x_{k+1}\right)-2 f\left(x_{k}\right)+f\left(x_{k-1}\right)}{h^{2}} \\
& +O\left(h^{2}\right) .
\end{aligned}
$$


Substitution of these approximations in (3.15) and (3.16) and a comparison of coefficients yields for the derivative:

$$
\begin{aligned}
\delta_{i}^{\prime} & =\frac{\gamma}{2 h^{2}} \text { for } i=k-1, \\
& =\frac{1-\gamma}{2 h^{2}} \text { for } i=k, \\
& =-\frac{\gamma}{2 h^{2}} \text { for } i=k+1, \\
& =\frac{\gamma-1}{2 h^{2}} \text { for } i=k+2, \\
& =0 \text { everywhere else } .
\end{aligned}
$$

For the second derivative:

$$
\begin{aligned}
\delta_{i}^{\prime \prime} & =\frac{\gamma}{h^{3}} \text { for } i=k-1, \\
& =\frac{1-3 \gamma}{h^{3}} \text { for } i=k, \\
& =\frac{3 \gamma-2}{h^{3}} \text { for } i=k+1, \\
& =\frac{1-\gamma}{h^{3}} \text { for } i=k+2, \\
& =0 \text { everywhere else } .
\end{aligned}
$$

Notice that we need four points to represent the derivatives of the delta function in this scheme.

\section{B. A multiple point delta function}

The procedure described in Sec. IIIA can be extended to represent the delta function over a larger number of points. On the one hand, this spreads out the delta, moving us away from our ideal of a function that is nonzero in as small a region as possible; on the other hand, it allows us to represent it more smoothly on our grid. The number of points $(2 n+2)$ that we use can thus be considered an optimization parameter, allowing us to trade localization for smoothness. As we shall see, there is typically a value of $n$ that represents a very good compromise.

We start off with the 'linear hat' delta function defined in 23] and 24]

$$
\delta_{i}=\left\{\begin{array}{ll}
\gamma_{i} / h & \text { for }\left|x_{i}-\alpha\right| \leq \epsilon=n h \\
0 & \text { otherwise }
\end{array},\right.
$$

where

$$
\gamma_{i}=\frac{1}{n}\left(1-\frac{\left|x_{i}-\alpha\right|}{n h}\right)
$$

and $n$ is an integer. Note that when $n=1, \gamma_{i}$ reduces to the $\gamma$ that was defined in Sec. IIIA Note also that $\gamma_{i}$ is non-zero only for $i \in[k, \ldots, k+2 n-1]$ (so that there are a total of $2 n$ points), and that $\alpha$ lies between the grid points $x_{k+n-1}$ and $x_{k+n}$. In this labeling scheme, $x_{k}$ is the smallest gridpoint where $\delta_{i}$ is nonzero.
Substituting this form of the delta function into our defining integral relation,

$$
h \sum_{i} f\left(x_{i}\right) \delta_{i} \simeq f(\alpha),
$$

we find

$$
\sum_{i} f\left(x_{i}\right) \gamma_{i} \simeq f(\alpha)
$$

The quantity $\gamma_{i}$ is thus a weighting factor whose weight depends on the distance of $x_{i}$ from $\alpha$. Setting $f(x)=1$, we find the property

$$
\sum_{i} \gamma_{i}=1
$$

Now consider the derivative of the delta function. Our goal is again to enforce the rule

$$
h \sum \delta_{i}^{\prime} f\left(x_{i}\right) \simeq-f^{\prime}(\alpha) \simeq-h \sum \delta_{i} f^{\prime}\left(x_{i}\right) .
$$

Inserting the finite difference formulae for the derivatives of $f$, Eqs. (3.17) and (3.18), into this relation, we find

$$
\begin{aligned}
f^{\prime}(\alpha) \simeq & -\sum_{i=k}^{k+2 n-1} \gamma_{i}\left[\frac{f\left(x_{i+1}\right)-f\left(x_{i-1}\right)}{2 h}\right] \\
= & -\frac{1}{2 h}\left[\left(\sum_{i=k+1}^{k+2 n-2} \gamma_{i-1} f\left(x_{i}\right)\right)\right] \\
& +\frac{1}{2 h}\left[\gamma_{k+2 n-2} f\left(x_{k+2 n-1}\right)\right] \\
& +\frac{1}{2 h}\left[\gamma_{k+2 n-1} f\left(x_{k+2 n}\right)\right] \\
& -\frac{1}{2 h}\left[\left(\sum_{i=k+1}^{k+2 n-2} \gamma_{i+1} f\left(x_{i}\right)\right)\right] \\
& +\frac{1}{2 h}\left[-\gamma_{k} f\left(x_{k-1}\right)-\gamma_{k+1} f\left(x_{k}\right)\right] .
\end{aligned}
$$

Comparing coefficients, we read off

$$
\begin{aligned}
\delta_{k-1}^{\prime}= & \frac{\gamma_{k}}{2 h^{2}}, \\
\delta_{k}^{\prime}= & \frac{\gamma_{k+1}}{2 h^{2}}, \\
\delta_{k+j}^{\prime}= & -\frac{\gamma_{k+j-1}-\gamma_{k+j+1}}{2 h^{2}} \\
& \text { for } j \in[1,2 n-2], \\
\delta_{k+2 n-1}^{\prime}= & -\frac{\gamma_{k+2 n-2}}{2 h^{2}}, \\
\delta_{k+2 n}^{\prime}= & -\frac{\gamma_{k+2 n-1}}{2 h^{2}} .
\end{aligned}
$$

The formulas for the delta derivative coefficients can be understood intuitively. The $n$-point generalization approximates the delta function as an isoceles triangle 
centered at $\alpha$ and sampled at $2 n$ points. The derivative is simply the slope of this isoceles triangle at all points, except at the center and the edges, where the derivative is discontinuous. The discontinuity is replaced by coefficients that ensure the integral properties of the derivative. The delta derivative takes a particularly simple formula in the "bulk":

$$
\begin{aligned}
\delta_{k+j}^{\prime} & =-\frac{\gamma_{k+j-1}-\gamma_{k+j+1}}{2 h^{2}} \text { for } j \in[1,2 n-2] \\
& =\frac{1}{2 n h^{2}}\left[\frac{\left|x_{k+j-1}-\alpha\right|}{n h}-\frac{\left|x_{k+j+1}-\alpha\right|}{n h}\right] \\
& =\left\{\begin{array}{ll}
\frac{1}{2 n^{2} h^{2}} & \text { for } x_{k+j+1}<\alpha-h \\
-\frac{1}{2 n^{2} h^{2}} & \text { for } x_{k+j+1}>\alpha+h
\end{array} .\right.
\end{aligned}
$$

Notice that the delta derivative coefficients are non-zero for $i \in[k-1, k+2 n]$ — one point wider in each direction than the span of the delta on the grid.

A similar analysis can be done for the second derivatives. We start off with

$$
\begin{aligned}
f^{\prime \prime}(\alpha) & \simeq \sum_{i=k}^{k+2 n-1} \gamma_{i} f^{\prime \prime}(x) \\
\left.h \sum_{i} \delta^{\prime \prime} f^{(} x_{i}\right) & =\sum_{i=k}^{k+2 n-1} \gamma_{i}\left[\frac{f\left(x_{i+1}\right)-2 f\left(x_{i}\right)+f\left(x_{i-1}\right)}{h^{2}}\right] .
\end{aligned}
$$

Reading off the coefficients leaves us with

$$
\begin{aligned}
\delta_{k-1}^{\prime \prime}= & \frac{\gamma_{k}}{h^{3}}, \\
\delta_{k}^{\prime \prime}= & \frac{\gamma_{k+1}-2 \gamma_{k}}{h^{3}}, \\
\delta_{k+j}^{\prime \prime}= & \frac{\gamma_{k+j+1}-2 \gamma_{k+j}+\gamma_{k+j-1}}{h^{3}}=0 \\
\delta_{k+2 n-1}^{\prime \prime}= & \frac{\gamma_{k+2 n-2}-2 \gamma_{k+2 n-1}}{h^{3}}, \\
\delta_{k+2 n}^{\prime \prime}= & \frac{\gamma_{k+2 n-1}}{h^{3}} .
\end{aligned}
$$

Notice that the second derivative is zero in the "bulk" - it corresponds to the second derivative of a line, with constant slope. Like the first derivative, these coefficients are non-zero for $i \in[k-1, k+2 n]$ — two points broader than the delta itself.

We have found that a very sharp delta function, like the two-point model described in the previous section, leads to instabilities for orbits with varying $r$ or $\theta$ (e.g., for eccentric orbits). Using a smoother $n$-point representation suppresses these instabilities; this will be discussed in greater detail in [31]. Since $r$ and $\theta$ are constant for circular, equatorial orbits, these instabilities do not arise for the cases examined in detail here. Thus for the case at hand, our numerical errors originating from the finite representation of the delta are smallest when $n=1$. This is demonstrated in detail in Sec. IV.

\section{Higher order interpolation for smoothness}

Using cubic interpolation (which requires a total of four points), we find the rule

$$
\begin{aligned}
h \sum_{i} f\left(x_{i}\right) \delta_{i}= & f(\alpha) \\
\Rightarrow h \sum_{i} f\left(x_{i}\right) \delta_{i}= & -\frac{\left(\alpha-x_{k+1}\right)\left(\alpha-x_{k+2}\right)\left(\alpha-x_{k+3}\right)}{6 h^{3}} f\left(x_{k}\right) \\
& +\frac{\left(\alpha-x_{k}\right)\left(\alpha-x_{k+2}\right)\left(\alpha-x_{k+3}\right)}{2 h^{3}} f\left(x_{k+1}\right) \\
& -\frac{\left(\alpha-x_{k}\right)\left(\alpha-x_{k+1}\right)\left(\alpha-x_{k+3}\right)}{2 h^{3}} f\left(x_{k+2}\right) \\
& +\frac{\left(\alpha-x_{k}\right)\left(\alpha-x_{k+1}\right)\left(\alpha-x_{k+2}\right)}{6 h^{3}} f\left(x_{k+3}\right) .
\end{aligned}
$$


The location $\alpha$ lies between grid points $x_{k+1}$ and $x_{k+2}$. From this expression, we read off the coefficients

$$
\begin{aligned}
\delta_{i} & =-\frac{\left(\alpha-x_{k+1}\right)\left(\alpha-x_{k+2}\right)\left(\alpha-x_{k+3}\right)}{6 h^{4}} \text { at } x_{k}, \\
& =+\frac{\left(\alpha-x_{k}\right)\left(\alpha-x_{k+2}\right)\left(\alpha-x_{k+3}\right)}{2 h^{4}} \text { at } x_{k+1}, \\
& =-\frac{\left(\alpha-x_{k}\right)\left(\alpha-x_{k+1}\right)\left(\alpha-x_{k+3}\right)}{2 h^{4}} \text { at } x_{k+2}, \\
& =+\frac{\left(\alpha-x_{k}\right)\left(\alpha-x_{k+1}\right)\left(\alpha-x_{k+2}\right)}{6 h^{4}} \text { at } x_{k+3} .
\end{aligned}
$$

A similar analysis for the first derivatives yields

$$
\begin{aligned}
\delta_{i}^{\prime} & =\frac{\left(x_{k+1}-\alpha\right)\left(\alpha-x_{k+2}\right)\left(\alpha-x_{k+3}\right)}{12 h^{5}} \text { at } x_{k-1}, \\
& =\frac{\left(\alpha-x_{k}\right)\left(\alpha-x_{k+2}\right)\left(\alpha-x_{k+3}\right)}{4 h^{5}} \text { at } x_{k}, \\
& =-\frac{\left(\alpha-x_{k+1}\right)\left(2 \alpha-3 x_{k}+x_{k+2}\right)\left(\alpha-x_{k+3}\right)}{12 h^{5}} \text { at } x_{k+1}, \\
& =-\frac{\left(\alpha-x_{k}\right)\left(\alpha-x_{k+2}\right)\left(2 \alpha+x_{k+1}-3 x_{k+3}\right)}{12 h^{5}} \text { at } x_{k+2}, \\
& =\frac{\left(\alpha-x_{k}\right)\left(\alpha-x_{k+1}\right)\left(\alpha-x_{k+3}\right)}{4 h^{5}} \text { at } x_{k+3}, \\
& =-\frac{\left(\alpha-x_{k}\right)\left(\alpha-x_{k+1}\right)\left(\alpha-x_{k+2}\right)}{12 h^{5}} \text { at } x_{k+4} .
\end{aligned}
$$

Note, we have used the second order finite difference formula, Eq. (3.17), to derive this result. In principle, higher order formulas for the derivative could have been used. We kept to the second order formula in order to keep the derivative stencil narrow, and also for consistency with our time-stepping algorithm.

Finally, for the second derivatives we find

$$
\begin{aligned}
\delta_{i}^{\prime \prime} & =\frac{\left(x_{k+1}-\alpha\right)\left(\alpha-x_{k+2}\right)\left(\alpha-x_{k+3}\right)}{6 h^{6}} \text { at } x_{k-1} \\
& =\frac{\left(5 \alpha-3 x_{k}-2 x_{k+1}\right)\left(\alpha-x_{k+2}\right)\left(\alpha-x_{k+3}\right)}{6 h^{6}} \text { at } x_{k} \\
& =-\frac{\left(10 \alpha^{2}-\left(9 x_{k}+4 x_{k+1}+7 x_{k+2}\right) \alpha+x_{k+1} x_{k+2}+3 x_{k}\left(x_{k+1}+2 x_{k+2}\right)\right)\left(\alpha-x_{k+3}\right)}{6 h^{6}} \text { at } x_{k+1} \\
& =\frac{\left(\alpha-x_{k}\right)\left(10 \alpha^{2}-\left(7 x_{k+1}+4 x_{k+2}+9 x_{k+3}\right) \alpha+3 x_{k+2} x_{k+3}+x_{k+1}\left(x_{k+2}+6 x_{k+3}\right)\right)}{6 h^{6}} \text { at } x_{k+2} \\
& =-\frac{\left(\alpha-x_{k}\right)\left(\alpha-x_{k+1}\right)\left(5 \alpha-2 x_{k+2}-3 x_{k+3}\right)}{6 h^{6}} \text { at } x_{k+3} \\
& =\frac{\left(\alpha-x_{k}\right)\left(\alpha-x_{k+1}\right)\left(\alpha-x_{k+2}\right)}{6 h^{6}} \text { at } x_{k+4} .
\end{aligned}
$$

As discussed in more detail in the following section, our analysis suggests that this cubic interpolation method works best.

We emphasize at this point that, although we are motivated by Teukolsky equation applications, our discussion here was not specialized to the Teukolsky equation in any way. The delta models sketched here can be used in any finite-difference numerical algorithm. We also note that one does not need to stop at cubic-order interpolation; the basic idea of that scheme could easily be extended to higher order if the application warranted it. As the order is increased, the "stencil" of the delta is likewise increased, pushing us away from the intuitive notion of a structureless impulse. This leads us to believe that there may be a certain interpolation order beyond which the model ceases to work well.

\section{Convergence with the discrete delta function}

The non-smooth nature of the discrete delta function makes understanding the convergence properties of 
a code based on this function somewhat subtle. Here we briefly summarize some key issues related to convergence with the discrete delta. This summary is based on detailed discussion of discretization errors given in Ref. [24]. The punchline of this discussion is that the discrete delta function is typically at least second-order convergent, and thus we expect our code to likewise be second-order convergent.

Let $\delta_{i}$ be the discretized version of $\delta(x-\alpha)$ defined on a discrete grid $x_{i}$, let $h=x_{i+1}-x_{i}$ be the grid spacing, and let $\delta_{i}$ be non-zero at $x_{k}, x_{k+1}, \ldots, x_{k+2 n-1}$. The continuous variable $\alpha$ lies between $x_{k+n-1}$ and $x_{k+n}$. This allows us to define a parameter $\eta$ such that

$$
\alpha=x_{k+n-1}+\eta h .
$$

This quantity is a measure of how close $\alpha$ is to a grid point; clearly, $0 \leq \eta \leq 1$.

We now define the moments of the discrete delta by

$$
M_{r}(\delta, \alpha, h)=h \sum_{i=k}^{k+2 n-1} \delta_{i}\left(x_{i}-\alpha\right)^{r}
$$

where $r$ is an integer. In the continuum limit, this definition becomes

$$
\begin{aligned}
M_{r} & \rightarrow \int \delta(x-\alpha)(x-\alpha)^{r} d x, \\
& =1 \quad r=0 \\
& =0 \quad r>0 .
\end{aligned}
$$

A discrete representation will clearly have the correct zeroth moment; however, it will only have $M_{r>0}=0$ up to some maximum $r$. Reference [24] proves that, if $q$ is the lowest integer such that $M_{q} \neq 0$, then

$$
\left|f(\alpha)-h \sum \delta_{i} f\left(x_{i}\right)\right| \leq C h^{q}
$$

where $C$ is approximately ${ }^{3}$ a constant. This delta representation is then $q$ th-order convergent.

For the multiple point discrete delta discussed in Sec. IIIB, we find $M_{0}=1, M_{1}=0, M_{2} \neq 0$. When we use this discrete delta, we therefore expect our code to be second-order convergent. We demonstrate this behavior in Sec. IVB. For the cubic delta function, we find $M_{0}=1, M_{1,2,3}=0, M_{4} \neq 0$. In this case, errors due to the delta representation are expected to be fourth order. However, since our stepping algorithm is itself secondorder, we expect second-order convergence overall.

\footnotetext{
${ }^{3}$ In our application, $C$ varies slightly depending on how close the delta peak is to a grid point.
}

\section{NUMERICAL IMPLEMENTATION AND EVALUATION OF THE DISCRETE DELTA FUNCTION}

We now implement the Teukolsky equation's source term using the techniques discussed in Sec. III for the simple case of a point particle in a circular, equatorial orbit around a massive black hole. Our goal is to compare the different forms of the discrete delta discussed in the previous section and to evaluate which is likely to work best for practical modeling of radiation from astrophysical systems. We also compare our discrete delta model to the Gaussian approximation that has been used in previous work, illustrating the power of this new model.

We obviously require some "standard" to compare our results against. Frequency-domain codes provide extremely accurate results for circular, equatorial orbits, largely since their emitted radiation is concentrated in a small number of multipoles; as such, they make an excellent standard against which to compare our results. We use the code described in [27] as our standard.

Tables \and I shows energy fluxes obtained from our code for the most dominant azimuthal modes, $|m|=2$ and 3 respectively. We compare these figures with those obtained from the code used in $[27]^{4}$.

There are two major reasons that the fluxes we compute depart from those computed by frequency-domain codes. First, the time-domain code must extract fluxes at some finite radius. The FD approach produces, by construction, the waveforms and fluxes that would be measured infinitely far from the generating binary; this simply cannot be done on a finite radial grid. A detailed discussion of the impact of finite extraction radius is given in Sec. V] In brief, we find by varying the extraction radius that fluxes can be fit to a very simple power law. This power law then allows us to infer the flux that would be measured by distant observers. The second source of error is simply numerical - finite difference errors plus the approximate nature of our discrete delta. Roughly speaking, accounting for finite extraction radius reduces our errors by about a factor of $2-5$; the residual error is thus most likely simply numerical error. This is described in much greater detail in Sec. $\nabla$.

Figures 2 and 3 illustrate a typical example of the structure for the Teukolsky function $\Psi$ that we find. We show the $m=2$ mode of an orbit with radius $r_{0}=7.9456 M$ around a Schwarzschild black hole; this orbit was selected in order to compare with results published in [20]. Note that the orbital period at this radius is $T=2 \pi \sqrt{r_{0}^{3} / M} \simeq 140 M$. The data is read out at radius $R \equiv r_{\text {extract }}=250 M, \theta=\pi / 2$; our numerical

\footnotetext{
${ }^{4}$ Symmetry in the azimuthal direction results in equal fluxes for $+m$ and $-m$ modes. Thus, $|m|$ refers to the sum of the fluxes from the $+m$ and $-m$ modes which is equal to twice the flux from either the $+m$ or the $-m$ mode.
} 
TABLE I: Energy flux extracted at $R \equiv r_{\text {extract }}=250 M$ for circular, equatorial orbits for the $m=|2|$ mode of a particle with mass $\mu / M=1 . a / M$ is the $\mathrm{BH}$ spin, $r_{0} / M$ is the orbital radius. The labels " $\delta$ " and "G" refer to the results from $\delta$-code and G-code respectively. Values listed under "FD" are the corresponding fluxes from the frequency-domain code used in [27].

\begin{tabular}{c|c|c|c|c|c|c|}
\hline \hline$a / M$ & $r_{0} / M$ & $\dot{E}_{\delta}$ & $\dot{E}_{F D}$ & $\left(\dot{E}_{\delta}-\dot{E}_{F D}\right) / \dot{E}_{F D}$ & $\left(\dot{E}_{G}-\dot{E}_{F D}\right) / \dot{E}_{F D}$ \\
\hline 0 & 6 & $7.385 \times 10^{-4}$ & $7.368 \times 10^{-4}$ & 0.0023 & $7.246 \times 10^{-4}$ & -0.017 \\
0 & 8 & $1.650 \times 10^{-4}$ & $1.651 \times 10^{-4}$ & -0.0055 & $1.623 \times 10^{-4}$ & -0.016 \\
0 & 10 & $5.344 \times 10^{-5}$ & $5.374 \times 10^{-5}$ & -0.0004 & $5.290 \times 10^{-5}$ & -0.016 \\
\hline 0.5 & 6 & $5.551 \times 10^{-4}$ & $5.539 \times 10^{-4}$ & 0.0022 & $5.437 \times 10^{-4}$ & -0.018 \\
0.5 & 8 & $1.399 \times 10^{-4}$ & $1.401 \times 10^{-4}$ & -0.0015 & $1.375 \times 10^{-4}$ & -0.019 \\
0.5 & 10 & $4.781 \times 10^{-5}$ & $4.812 \times 10^{-5}$ & -0.0065 & $4.691 \times 10^{-5}$ & -0.025 \\
\hline 0.9 & 4 & $2.654 \times 10^{-3}$ & $2.662 \times 10^{-3}$ & -0.0030 & $2.611 \times 10^{-3}$ & -0.019 \\
0.9 & 6 & $4.614 \times 10^{-4}$ & $4.621 \times 10^{-4}$ & -0.0016 & $4.531 \times 10^{-4}$ & -0.020 \\
0.9 & 8 & $1.249 \times 10^{-4}$ & $1.254 \times 10^{-4}$ & -0.0039 & $1.230 \times 10^{-4}$ & -0.019 \\
0.9 & 10 & $4.419 \times 10^{-5}$ & $4.456 \times 10^{-5}$ & -0.0084 & $4.339 \times 10^{-5}$ & -0.026 \\
\hline 0.99 & 4 & $2.469 \times 10^{-3}$ & $2.484 \times 10^{-3}$ & -0.0059 & $2.434 \times 10^{-3}$ & -0.020 \\
0.99 & 6 & $4.450 \times 10^{-4}$ & $4.461 \times 10^{-4}$ & -0.0024 & $4.372 \times 10^{-4}$ & -0.020 \\
0.99 & 8 & $1.221 \times 10^{-4}$ & $1.226 \times 10^{-4}$ & -0.0041 & $1.201 \times 10^{-4}$ & -0.020 \\
0.99 & 10 & $4.346 \times 10^{-5}$ & $4.386 \times 10^{-5}$ & -0.0090 & $4.270 \times 10^{-5}$ & -0.026 \\
\hline \hline
\end{tabular}

TABLE II: Energy flux extracted at $250 M$ for circular, equatorial orbits for the $m=|3|$ mode of a particle with mass $\mu / M=1$. All symbols and notation are as in Table@

\begin{tabular}{c|c|c|c|c|c|c|}
\hline \hline$a / M$ & $r_{0} / M$ & $\dot{E}_{\delta}$ & $\dot{E}_{F D}$ & $\left(\dot{E}_{\delta}-\dot{E}_{F D}\right) / \dot{E}_{F D}$ & $\left(\dot{E}_{G}-\dot{E}_{F D}\right) / \dot{E}_{F D}$ \\
\hline 0 & 6 & $1.465 \times 10^{-4}$ & $1.460 \times 10^{-4}$ & 0.0035 & $1.431 \times 10^{-4}$ & -0.020 \\
0 & 8 & $2.445 \times 10^{-5}$ & $2.449 \times 10^{-5}$ & -0.0017 & $2.399 \times 10^{-5}$ & -0.020 \\
0 & 10 & $6.383 \times 10^{-6}$ & $6.435 \times 10^{-6}$ & -0.0080 & $6.291 \times 10^{-6}$ & -0.022 \\
\hline 0.5 & 6 & $1.015 \times 10^{-4}$ & $1.014 \times 10^{-4}$ & 0.0011 & $0.992 \times 10^{-4}$ & -0.021 \\
0.5 & 8 & $1.993 \times 10^{-5}$ & $1.980 \times 10^{-5}$ & 0.0066 & $1.935 \times 10^{-5}$ & -0.023 \\
0.5 & 10 & $5.521 \times 10^{-6}$ & $5.572 \times 10^{-6}$ & -0.0090 & $5.410 \times 10^{-6}$ & -0.029 \\
\hline 0.9 & 4 & $6.485 \times 10^{-4}$ & $6.467 \times 10^{-4}$ & 0.0028 & $6.336 \times 10^{-4}$ & -0.020 \\
0.9 & 6 & $8.031 \times 10^{-5}$ & $8.043 \times 10^{-5}$ & -0.0015 & $7.865 \times 10^{-5}$ & -0.022 \\
0.9 & 8 & $1.710 \times 10^{-5}$ & $1.717 \times 10^{-5}$ & -0.0043 & $1.677 \times 10^{-5}$ & -0.023 \\
0.9 & 10 & $4.992 \times 10^{-6}$ & $5.044 \times 10^{-6}$ & -0.0103 & $4.893 \times 10^{-6}$ & -0.030 \\
\hline 0.99 & 4 & $5.932 \times 10^{-4}$ & $5.924 \times 10^{-4}$ & 0.0014 & $5.805 \times 10^{-4}$ & -0.021 \\
0.99 & 6 & $7.688 \times 10^{-5}$ & $7.688 \times 10^{-5}$ & $-4.8 \times 10^{-5}$ & $7.528 \times 10^{-5}$ & -0.022 \\
0.99 & 8 & $1.6542 \times 10^{-5}$ & $1.669 \times 10^{-5}$ & -0.0086 & $1.628 \times 10^{-5}$ & -0.025 \\
0.99 & 10 & $4.879 \times 10^{-6}$ & $4.942 \times 10^{-6}$ & -0.0127 & $4.792 \times 10^{-6}$ & -0.030 \\
\hline \hline
\end{tabular}


grid runs from $-100 M \leq r^{*} \leq 500 M$, with a resolution $\delta r^{*}=0.0625 M$, and from $0 \leq \theta \leq \pi$ with $\delta \theta=\pi / 40$.

Figure 2 shows the real part of $\Psi$ over a broad span of time, from the beginning of our simulation to $t \simeq 800 \mathrm{M}$. At $t \sim 250 M$, a very high amplitude, unphysical burst of radiation reaches the extraction radius. This spurious burst is due to our initial conditions: We initially set $\Psi=0$ and $\partial_{t} \Psi=0$, which is not consistent with our source function. The time at which this burst reaches the extraction radius is perfectly consistent with radiation propagating at the speed of light $(c=1$ in our units) across our numerical grid. The burst quickly propagates off the grid, and the solution for $\Psi$ settles down to a simple oscillation. This is shown in Fig. 3. which zooms in on the behavior of $\Psi$ for $t \gtrsim 350 M$. Notice that $\Psi_{R}$ has an oscillation period of about $70 \mathrm{M}$, precisely what we expect for the $m=2$ mode of a source whose orbital frequency is $140 M$. The energy flux we find from this mode is $\dot{E} / \mu^{2}=1.708 \times 10^{-4}$, in excellent agreement with results published in Ref. [20] (compare Table II of [20], noting that our results require summing over all $l$ for fixed $m$ ).

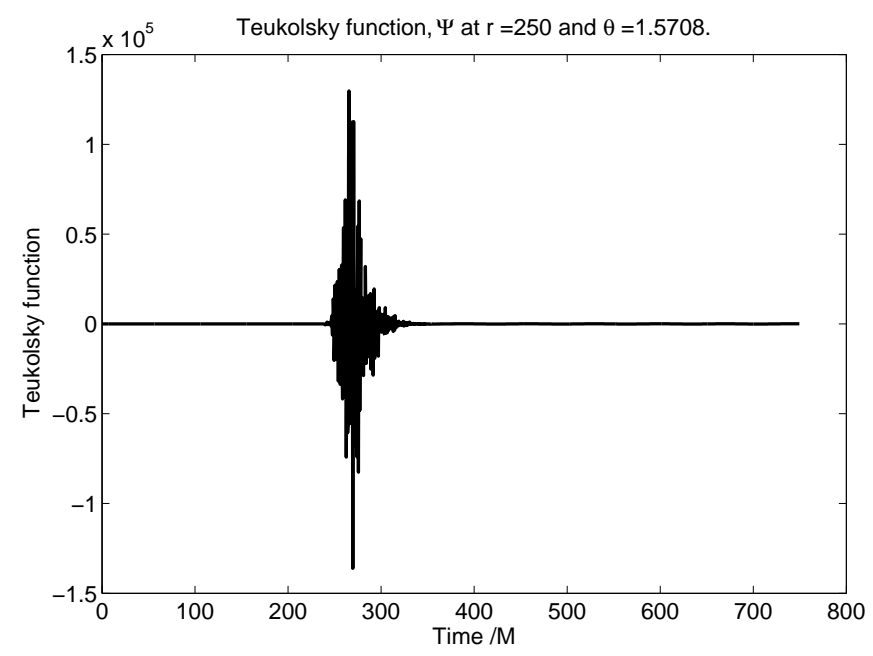

FIG. 2: The real part of the $m=2$ mode of the Teukolsky function $\Psi$ as a function of time for a point particle of mass $\mu / M=0.01$ in a circular orbit of radius $r_{0} / M=7.9456$. These data were extracted in the equatorial plane $(\theta=\pi / 2)$ at radius $R=250 M$. At this location the Teukolsky function is zero by construction until $t \simeq 250 M$, at which point a spurious burst reaches the extraction radius. This burst is due to our unphysical initial conditions; it quickly propagates off the grid, leaving a reasonable physical solution for all time afterwards.

Figure 4 illustrates the spatial behavior of the real part of $\Psi$ at a particular moment in time $(t=312 M)$. This plot illustrates the behavior of $\operatorname{Re} \Psi$ as a function $r^{*}$ and $\theta$ over a wide span of our grid. Clearly visible are the $m=2$ mode of the radiation propagating to large radius as well as the nearly singular delta function source itself.

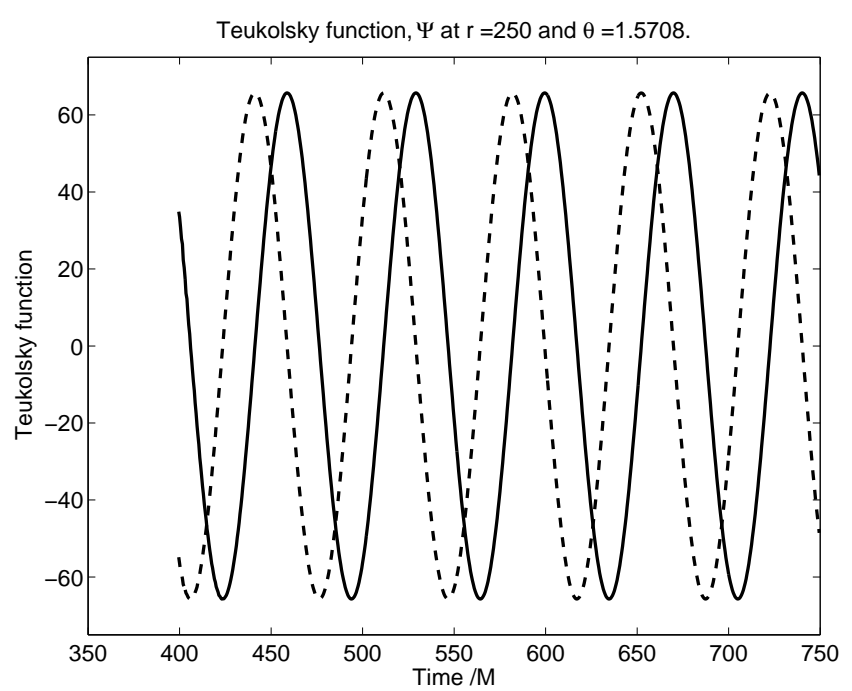

FIG. 3: The same as Fig. 2, but zooming in on the data for $t \gtrsim 350 M$. Solid and dashed lines are the real and imaginary parts of $\Psi$ respectively. The Teukolsky function oscillates with a period of about $70 M$; since the source has a period of about $140 M$, this is exactly what we expect for the $m=2$ mode. We measure the total flux of energy carried by this mode to be $\dot{E} / \mu^{2}=1.708 \times 10^{-4}$, in agreement with previous results (see, e.g., Ref. [20]).

\section{A. Comparison of different discrete delta functions}

In this section, we compare results from the various models for the delta function presented in Sec. III The variable point approximation for the linear delta function, presented in Sec. III provides us with a nice handle to study the convergence of our results. As we increase $n$, the half-width of the delta function, the singularity spreads out and its sharpness decreases. Notice that the physical spread of the source term is $2(n+1) \delta r^{*}$ (due to the spread of the delta derivatives). Thus, decreasing the resolution has the same effect on the physical width as decreasing $n$.

In Tables III and IV we present results describing two equatorial circular orbits, one in the extremely strong field $\left(r_{0} / M=2.32, a / M=0.9\right)$, and another at weaker field $\left(r_{0} / M=12, a / M=0\right)$. We show the variation in flux with $n$, the half-width of the radial delta function. The angular delta function is represented using two points (i.e., $n_{\text {ang }}=1$ ), the minimum number of nontrivial points. The resolutions $\left(\delta r_{*}, \delta \theta\right)$ are held fixed at $(0.0625 M, \pi / 40)$

The third and fourth columns of Tables III and IV compare the flux in energy carried by GWs as computed using the $\delta$-code to flux computed using our frequencydomain standard. The third column gives a "raw" comparison - we extract the time-domain fluxes at radius $R=250 M$ and compare to the frequency-domain result. In the fourth column, we extrapolate the time-domain data, $R \rightarrow \infty$, using the algorithm described in Sec. V. 


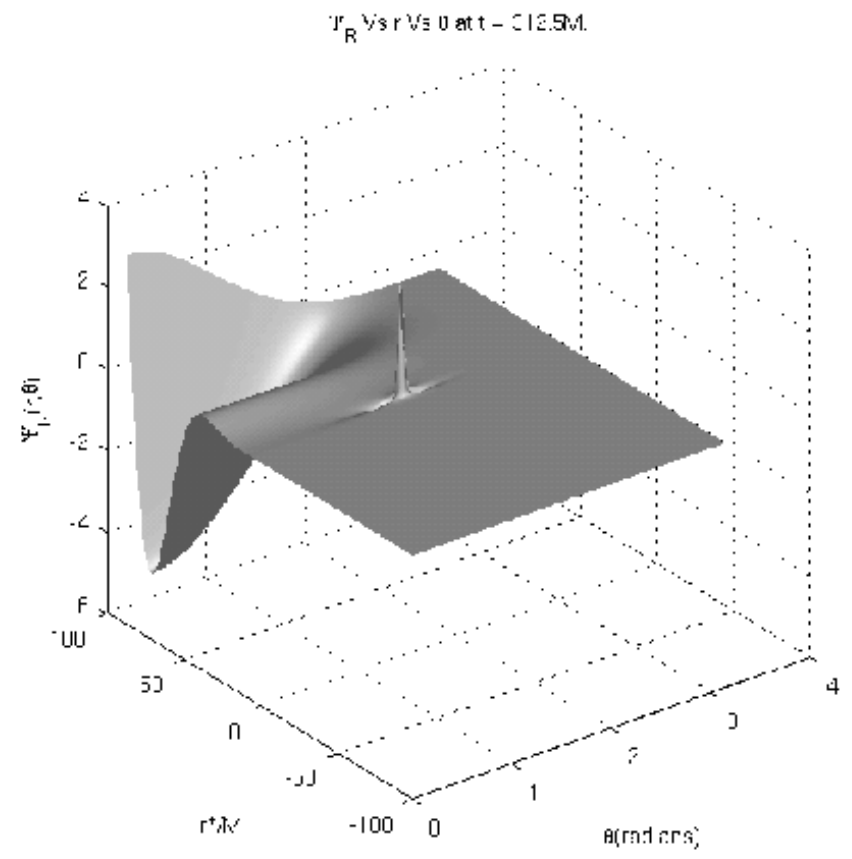

FIG. 4: The same as Fig. 2, but now showing the data for a given moment in time $(t=312.5 M)$ for a wide range of $r^{*}$ and $\theta$. Along with the outward propagating radiation packet visible at large radius, the nearly singular delta function source is clearly visible at the particle's position.

The fourth column thus contains the most relevant data for assessing which delta representation is "best". We include the third column to demonstrate that the difference before extrapolating is not terribly large, but that it is large enough that the gain due to this extrapolation is significant. It also illustrates that not performing the extrapolation can mislead regarding which form of the delta is most accurate.

Data from Table IV indicate that, among the $n$-point representations, $n=1$ gives the best results for circular, equatorial orbits. The cubic representation, however, is even better - the smoothness of this technique apparently reduces error even more. We choose the cubic delta for the remainder of our analysis because it is both smooth and accurate.

By contrast, the most accurate flux in Table III occurs when $n=7$ (total of 8 points to represent the source), rather than $n=1$. The reason is due to a competition between errors from finite differencing and errors from our delta representation. In particular, we have noticed experimentally that finite difference errors tend, on average, to spuriously decrease our measured flux; errors from spreading the delta over the grid tend to augment the flux. (We emphasize that this is merely a rule-ofthumb tendency we have noted; we also emphasize that we do not as of yet have a good explanation for these effects.)

As we approach the horizon, finite difference errors tend to become more important. This can be compensated by increasing the width of our delta representations. At $n=7$, the spread of our source is just enough to accurately compensate for finite difference errors. At larger radius (e.g., the $r_{0}=12 M$ orbit shown in Table IV], finite difference errors are so small that we do best using the minimum number of points possible in our model.

\section{B. Convergence of our code}

As discussed in Sec. IIID we generally expect a code built using the discrete delta on grid with spacing $h$ to exhibit $O\left(h^{2}\right)$ convergence. In particular, we expect the Weyl scalar $\psi_{4}$ to show second-order convergence. We check this expectation by examining the flux of energy carried by gravitational waves. Since we expect $\psi_{4}=$ $\psi_{4}^{\text {true }}+O\left(h^{2}\right)$, we likewise expect $\dot{E}$ to exhibit secondorder convergence:

$$
\dot{E} \sim\left|\psi_{4}\right|^{2} \sim\left|\psi_{4}^{\text {true }}\right|^{2}+O\left(h^{2}\right) .
$$

To demonstrate this convergence, we show the energy flux measured at $R=250 \mathrm{M}$ for two different strong-field ${ }^{5}$ orbits: $r_{0}=5 M, a=0.8 M$ and $r_{0}=4.64 M, a=0.9 M$. The radial and angular grids are set to

$$
\begin{array}{r}
\delta r^{*}=0.0625 \times 2^{-b / 4}, \\
\delta \theta=\pi / 30 \times 2^{-b / 4} .
\end{array}
$$

Actually, $\delta \theta$ was modified slightly from this to insure that $\theta=\pi / 2$ lies exactly on a grid point. This reduced variations about the main $h^{2}$ trend owing to the slight dependence of the proportionality "constant" on the delta's peak (cf. discussion in Sec. IIID). Figure 5 shows the results our runs for $b \in[-1, \ldots, 4]$. Convergence is shown by examining the fractional error with respect to our densest grid,

$$
\text { error } \equiv \frac{\left|\dot{E}_{b}-\dot{E}_{4}\right|}{\dot{E}_{4}}
$$

where $\dot{E}_{b}$ is the flux inferred at grid parameter $b$. We normalize to $b=4$ since it is the densest grid available to us. Modulo some slight oscillations, the overall trend of our data is in very good agreement with second-order convergence.

\footnotetext{
${ }^{5}$ It's worth noting that we found it to be rather difficult to demonstrate convergence using weak-field orbits. For such orbits, the differences in our computed fluxes were quite small as we varied our grid density. We need strong-field orbits in order for the errors to be large enough that the convergence trend is apparent.
} 
TABLE III: Comparison of several implementations of the discrete delta function. We show results for the linear hat delta described in Sec. IIIB, as well as the cubic delta function described in Sec. IIIC. All fluxes are measured at $R=250 M$ for the $|m|=2$ mode. For the linear hat delta, the total number of points in the function is $2(n+1)$. The cubic delta uses 6 points in all. These results are for orbits of radius $r_{0}=2.32 M$ about a black hole with $a=0.9 M$. The total flux in $|m|=2$ modes according to our frequency-domain standard is $\dot{E}_{\mathrm{FD}} / \mu^{2}=2.061 \times 10^{-2}$.

\begin{tabular}{|c|c|c|c|c|}
\hline Total points, $2(n+1)$ & $\dot{E}_{250}$ & $\left(\dot{E}_{250}-\dot{E}_{F D}\right) / \dot{E}_{F D}$ & $\dot{E}_{\infty}$ & $\left(\dot{E}_{\infty}-\dot{E}_{F D}\right) / \dot{E}_{F D}$ \\
\hline 64 & $2.889 \times 10^{-2}$ & $4.0 \times 10^{-1}$ & $2.890 \times 10^{-2}$ & $4.0 \times 10^{-1}$ \\
32 & $2.194 \times 10^{-2}$ & $6.5 \times 10^{-2}$ & $2.195 \times 10^{-2}$ & $6.5 \times 10^{-2}$ \\
16 & $2.055 \times 10^{-2}$ & $-2.8 \times 10^{-3}$ & $2.056 \times 10^{-2}$ & $-2.4 \times 10^{-3}$ \\
8 & $2.027 \times 10^{-2}$ & $-1.6 \times 10^{-2}$ & $2.028 \times 10^{-2}$ & $-1.6 \times 10^{-2}$ \\
4 & $2.023 \times 10^{-2}$ & $-1.9 \times 10^{-2}$ & $2.024 \times 10^{-2}$ & $-1.8 \times 10^{-2}$ \\
cubic & $2.024 \times 10^{-2}$ & $-1.8 \times 10^{-2}$ & $2.024 \times 10^{-2}$ & $-1.8 \times 10^{-2}$ \\
\hline
\end{tabular}

TABLE IV: Same as Table III but now for an orbit with $r_{0}=12 M$ about a black hole with $a=0$. The frequency-domain flux for $|m|=2$ modes in this case is $\dot{E}_{\mathrm{FD}} / \mu^{2}=2.172 \times 10^{-5}$.

\begin{tabular}{|c|c|c|c|c|}
\hline Total points, $2(n+1)$ & $\dot{E}_{250}$ & $\left(\dot{E}_{250}-\dot{E}_{F D}\right) / \dot{E}_{F D}$ & $\dot{E}_{\infty}$ & $\left(\dot{E}_{\infty}-\dot{E}_{F D}\right) / \dot{E}_{F D}$ \\
\hline 64 & $2.342 \times 10^{-5}$ & $7.8 \times 10^{-2}$ & $2.376 \times 10^{-5}$ & $9.4 \times 10^{-1}$ \\
32 & $2.191 \times 10^{-5}$ & $8.7 \times 10^{-3}$ & $2.224 \times 10^{-5}$ & $2.4 \times 10^{-1}$ \\
16 & $2.156 \times 10^{-5}$ & $-7.5 \times 10^{-3}$ & $2.187 \times 10^{-5}$ & $7.1 \times 10^{-3}$ \\
8 & $2.148 \times 10^{-5}$ & $-1.1 \times 10^{-2}$ & $2.179 \times 10^{-5}$ & $3.3 \times 10^{-3}$ \\
4 & $2.146 \times 10^{-5}$ & $-1.2 \times 10^{-2}$ & $2.177 \times 10^{-5}$ & $2.5 \times 10^{-3}$ \\
cubic & $2.145 \times 10^{-5}$ & $-1.2 \times 10^{-2}$ & $2.177 \times 10^{-5}$ & $2.3 \times 10^{-3}$ \\
\hline
\end{tabular}

\section{Comparison of discrete and Gaussian approximations for the numerical delta}

The work in 17, 18, 19] approximates the deltafunction by a narrow Gaussian such that it integrates to unity over the numerical grid. The Gaussian smears out the singularity and thus the source term is non zero at a large number of points on the numerical grid. In contrast, the models presented in Sec. III use only a few points to depict the delta-function and thus do not share this disadvantage. A comparison on the same hardware and software platform showed that the techniques used here (the $\delta$-code) are about twelve times faster than the ones that use a smeared Gaussian (the G-code). The last two columns in Tables [ and [1] show the fluxes from the Gaussian-approximated delta function. Note that the errors in these fluxes are about $2-3 \%$, quite a bit higher than errors from the $\delta$-code. Both codes were run with identical parameters and grid resolutions. The accuracy of both codes improves with higher resolution, and improvement in both is seen by moving the extraction radius farther out. However, when these parameters are fixed, we find that the $\delta$-code is faster and demonstrates higher accuracy.

\section{ACCOUNTING FOR FINITE EXTRACTION RADIUS}

When one discusses the gravitational-wave fluxes which a system generates, one is normally interested in their asymptotic value infinitely far away. It is of course not possible for a finite coordinate grid to reach all the way into this distant zone, so it is of great importance to understand how our fluxes vary with respect to our finite extraction radius $R$.

In flat spacetime, the extraction radius is not very important; it just needs to be sufficiently far away that the field it measures is purely radiative (i.e, not contaminated by near-field effects). Conservation laws guarantee that fluxes follow a $1 / r^{2}$ law in this region, and so the integrated flux is independent of extraction radius.

Things are not so simple in a curved spacetime radiation is effectively scattered off of spacetime curvature, modifying its propagation characteristics compared to flat spacetime intuition. This is responsible for the late time "tails" that are seen when a radiation packet propagates away from a black hole (cf. the late time behavior seen in Fig. (1). These tails can be regarded, heuristically, as radiation whose propagation to large radius was delayed by scattering off the spacetime. It also causes the integrated flux to depend on and vary with the radius at which it is measured.

We now examine how our fluxes vary with respect to

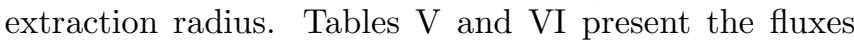
measured for four representative strong-field orbits. In each case, we measure $\dot{E}$ for the $|m|=2$ and $|m|=3$ modes at extraction radii $R / M=100,200,300,400$, 500 , and 600 . These data are then fit to the ansatz

$$
\dot{E}=\dot{E}_{\infty}\left[1-q\left(m \Omega_{\mathrm{orb}} R\right)^{-p}\right]
$$




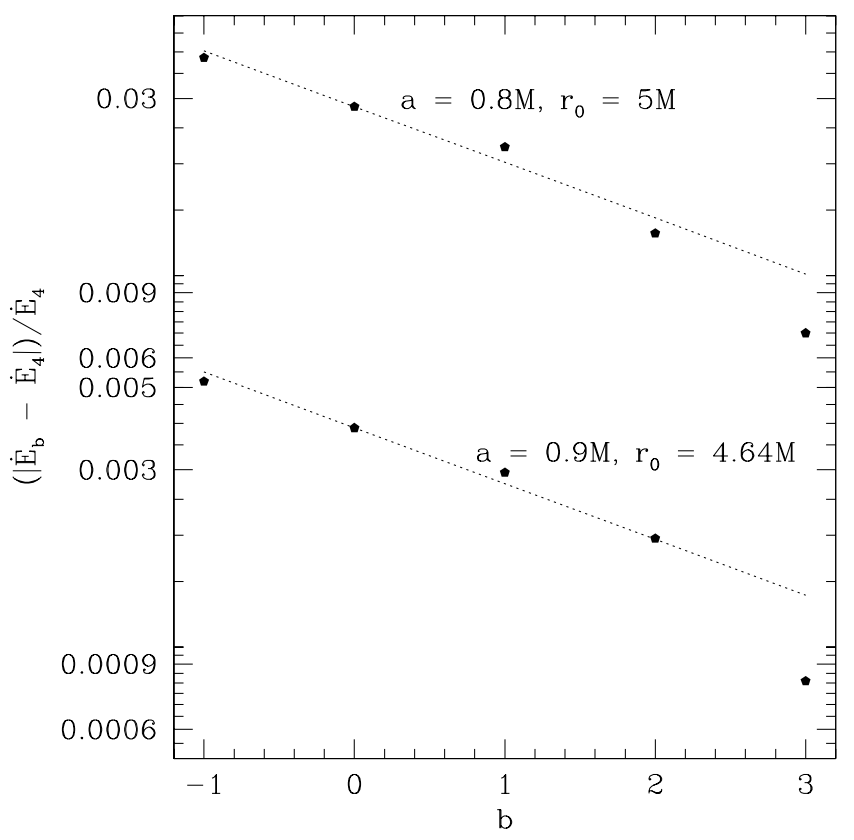

FIG. 5: An ilustration of our code's convergence behavior. We show the fractional deviation in energy flux in the $|m|=2$ mode, measured at $R=250 M$ as a function of grid spacing. The grid is controlled by the integer $b$ using $\delta r^{*}=0.0625 \times$ $2^{-b / 4}, \delta \theta=\pi / 30 \times 2^{-b / 4}$, with $b \in[-1, \ldots, 4]$. The upper data set is for fluxes measured from an orbit with $a=0.8 M$, $r_{0}=5 M$; the lower set is for $a=0.9 M, r_{0}=4.64 M$. For each data set, the dotted line represents what we would expect for perfect second-order convergence (fit arbitrarily to the data for $b=0$ ); the large dots represent our actual convergence data.

The parameters $q, p$, and $\dot{E}_{\infty}$ are determined by the fit. Notice that $\dot{E}_{\infty}$ represents the flux that (according to this ansatz) would be measured infinitely far away. Note that this form was suggested to us by L. M. Burko 34, and replaces a previous version which used $\left(r_{\text {orb }} / R\right)^{p}$ rather than $\left(m \Omega_{\mathrm{orb}} R\right)^{-p}$. The two forms can be easily related to one another; however, the form involving $m \Omega_{\text {orb }}$ emphasizes that it is the asymptotic behavior of the mode, rather than a property of the orbit, that sets $\dot{E}$. This form should also be more readily extendable to non-circular orbits.

Figure 5 shows an example of how well this fit works for one of the cases given in Table $\mathrm{V}\left(r_{0} / M=10\right.$, $a / M=0.99, m=2)$. Pragmatically, this ansatz appears to fit the data quite well; the quality shown in Fig. 6 is typical for the data that we examined. Interestingly, we find in all cases that the exponent $p \simeq 2$, independent of black hole spin, orbit radius, or mode number. Detailed calculations which we will present elsewhere 35] shows that this corresponds to the dominant correction to the asymptotic behavior of $\psi_{4}$. Such behavior was also demonstrated by Newman and Unti [36] (although our results do not currently agree with theirs when $a \neq 0$; we are investigating this discrepancy).

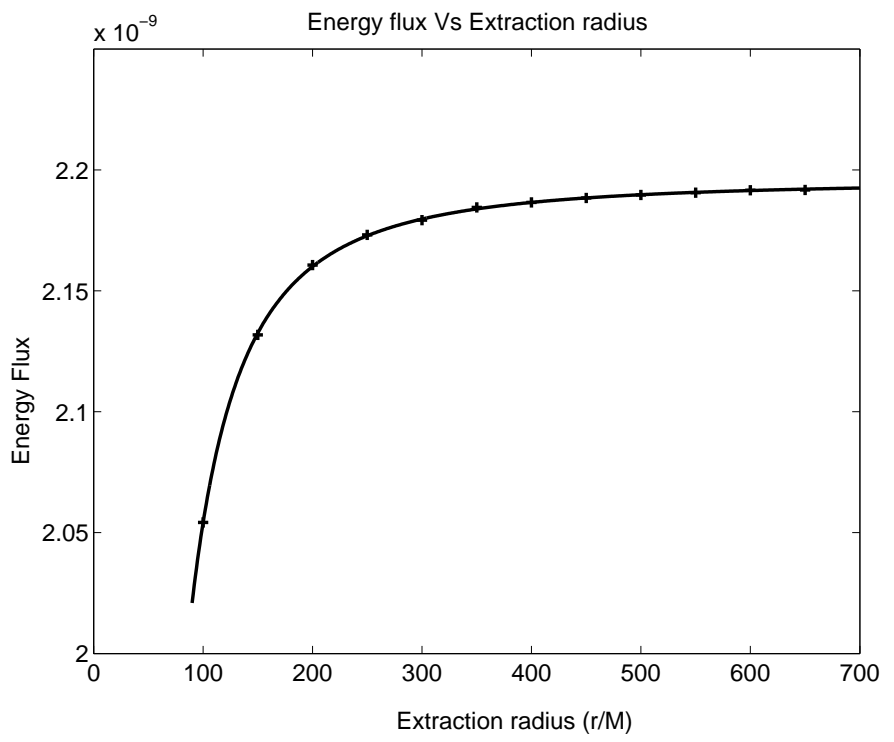

FIG. 6: A power law fit to our numerically extracted energy fluxes for the case $r_{0} / M=10, a / M=0.99, m=+2$. Numerical data is indicated by the dots; the curve is the best fit we obtain for the ansatz given by Eq. (5.1). For this case, the best fit parameters are $q=7.45, p=2.06, \dot{E}_{\infty}=2.197 \times 10^{-5}$.

We now use the fit (5.1) to compare the extrapolated measured flux $\dot{E}_{\infty}$ to frequency-domain results $\dot{E}_{\mathrm{FD}}$. This is shown in the last column of TableVI. In all cases, the error we find is less than $1 \%$, sometimes substantially less. A similar fit can be performed on the angular momentum flux, with similar results. We conclude that the fit (5.1) accounts for finite extraction radius, providing an accurate estimate for the fluxes that a particle radiates to infinity. Residual errors are thus much more likely to be true measures of numerical error in our calculation, and not an artifact of the extraction.

\section{SUMMARY AND FUTURE WORK}

We have presented a simple, new technique for modeling the Dirac delta function and its derivatives on a finite difference grid. This technique requires that the source be modeled only on a handful of points on the grid. Our particular goal in this analysis is to model a pointlike source function for the time-domain Teukolsky equation, appropriate to describe the smaller member of an extreme mass ratio binary. We emphasize that our models for the discrete delta and its derivatives are more broadly applicable than just the Teukolsky equation these techniques can be used in any context that requires modeling a sharp, delta-like function on a finite difference grid. 
TABLE V: Fluxes extracted at a sequence of radii on the numerical grid. $a / M$ is the $\mathrm{BH}$ spin, $r_{0} / M$ is the orbital radius and $|m|$ is the azimuthal mode. $\dot{E}_{R}$ is the flux measured at radius $R M$.

\begin{tabular}{|c|c|c|c|c|c|c|c|c|}
\hline \hline$|m|$ & $a / M$ & $r_{0} / M$ & $\dot{E}_{100}$ & $\dot{E}_{200}$ & $\dot{E}_{300}$ & $\dot{E}_{400}$ & $\dot{E}_{500}$ & $\dot{E}_{600}$ \\
\hline 2 & 0.99 & 4 & $2.4567 \times 10^{-3}$ & $2.4681 \times 10^{-3}$ & $2.4702 \times 10^{-3}$ & $2.4709 \times 10^{-3}$ & $2.4712 \times 10^{-3}$ & $2.4714 \times 10^{-3}$ \\
2 & 0.99 & 10 & $4.1032 \times 10^{-5}$ & $4.3209 \times 10^{-5}$ & $4.3598 \times 10^{-5}$ & $4.3767 \times 10^{-5}$ & $4.3828 \times 10^{-5}$ & $4.3861 \times 10^{-5}$ \\
2 & 0.90 & 10 & $4.1729 \times 10^{-5}$ & $4.3930 \times 10^{-5}$ & $4.4322 \times 10^{-5}$ & $4.4456 \times 10^{-5}$ & $4.4517 \times 10^{-5}$ & $4.4550 \times 10^{-5}$ \\
2 & 0.00 & 12 & $1.9584 \times 10^{-5}$ & $2.1256 \times 10^{-5}$ & $2.1554 \times 10^{-5}$ & $2.1654 \times 10^{-5}$ & $2.1699 \times 10^{-5}$ & $2.1723 \times 10^{-5}$ \\
\hline 3 & 0.99 & 4 & $5.8962 \times 10^{-4}$ & $5.9278 \times 10^{-4}$ & $5.9334 \times 10^{-4}$ & $5.9353 \times 10^{-4}$ & $5.9361 \times 10^{-4}$ & $5.9364 \times 10^{-4}$ \\
3 & 0.99 & 10 & $4.5778 \times 10^{-6}$ & $4.8558 \times 10^{-6}$ & $4.9051 \times 10^{-6}$ & $4.9220 \times 10^{-6}$ & $4.9297 \times 10^{-6}$ & $4.9339 \times 10^{-6}$ \\
3 & 0.90 & 10 & $4.6791 \times 10^{-6}$ & $4.9588 \times 10^{-6}$ & $5.0085 \times 10^{-6}$ & $5.0255 \times 10^{-6}$ & $5.0333 \times 10^{-6}$ & $5.0375 \times 10^{-6}$ \\
3 & 0.00 & 12 & $1.9528 \times 10^{-6}$ & $2.1326 \times 10^{-6}$ & $2.1650 \times 10^{-6}$ & $2.1761 \times 10^{-6}$ & $2.1812 \times 10^{-6}$ & $2.1839 \times 10^{-6}$ \\
\hline \hline
\end{tabular}

TABLE VI: Best fit parameters, $\dot{E}_{\infty}, p, q$ [appearing in Eq. [5.1)] for data presented in Table $\nabla$

\begin{tabular}{|c|c|c|c|c|c|c|c|}
\hline$|m|$ & $a / M$ & $r_{0} / M$ & $\dot{E}_{\infty}$ & $p$ & $q$ & $\dot{E}_{F D}$ & $\left(\dot{E}_{\infty}-\dot{E}_{F D}\right) / \dot{E}_{F D}$ \\
\hline 2 & 0.99 & 4 & $2.4718 \times 10^{-3}$ & 2.04 & 3.40 & $2.4836 \times 10^{-3}$ & -0.0048 \\
\hline 2 & 0.99 & 10 & $4.3953 \times 10^{-5}$ & 1.96 & 2.31 & $4.3948 \times 10^{-5}$ & 0.0001 \\
\hline 2 & 0.90 & 10 & $4.462 \times 10^{-5}$ & 2.05 & 2.70 & $4.4560 \times 10^{-5}$ & 0.0014 \\
\hline 2 & 0.00 & 12 & $2.1779 \times 10^{-5}$ & 2.07 & 2.59 & $2.1722 \times 10^{-5}$ & 0.0026 \\
\hline 3 & 0.99 & 4 & $5.9375 \times 10^{-4}$ & 2.09 & 10.74 & $5.9239 \times 10^{-4}$ & 0.0023 \\
\hline 3 & 0.99 & 10 & $4.9428 \times 10^{-6}$ & 2.06 & 7.27 & $4.9417 \times 10^{-6}$ & 0.0002 \\
\hline 3 & 0.90 & 10 & $5.0466 \times 10^{-6}$ & 2.06 & 7.16 & $5.0440 \times 10^{-6}$ & 0.0005 \\
\hline 3 & 0.00 & 12 & $2.1900 \times 10^{-6}$ & 2.05 & 6.20 & $2.1890 \times 10^{-6}$ & 0.0004 \\
\hline
\end{tabular}

We test this approach by solving the Teukolsky equation for a test body in a circular, equatorial orbit of a Kerr black hole. Comparing with a well tested timedomain code that treats the orbiting body using a truncated Gaussian, we find that this new approach is extremely fast (often by a factor of $\sim 10$ ) and accurate. Using a frequency-domain code as a benchmark to compare the flux of energy carried by gravitational waves, we find that the code which uses the discrete delta function is typically a factor of $2-5$ more accurate than the Gaussian treatment most commonly used previously. This accuracy can be improved still further (at least for fluxes) by using a simple fit that accounts for the variation of the flux with the extraction radius. Combining our new source function with this fitting law, we find that our code agrees with the frequency-domain benchmark with errors smaller than $1 \%$ for a large fraction of parameter space, sometimes significantly smaller.

Since the goal of this analysis is to contribute to the modeling of EMRI gravitational-wave sources, the restriction to circular and equatorial orbits, though a useful, illustrative test, is not astrophysically realistic. Since such binaries form through scattering processes, they are expected to have substantial eccentricity [4], and the secondary's orbit should have no special alignment with the spin axis of the large black hole. Our next analysis will study how well this new technique handles such orbits.
This realistic case is substantially more difficult to treat than circular, equatorial orbits, since the orbiting body (and our discrete delta model) very rapidly crosses back and forth over gridpoints in both the radial and latitudinal directions. As this paper is being completed, this work is well underway. Early analysis points to excellent results - we get very good results even when we move our discrete delta model rapidly in a dynamical orbit. We also plan to examine wave emission from nongeodesic trajectories (in preparation for using the code to calculate wave emission from an inspiral sequence), and to examine plunging orbits (with a plan to examine waves from the transition between late inspiral to final plunge [37]).

The final goal of this work will be to compute adiabatic inspiral waveforms using a hybrid of frequencydomain and time-domain, as described in the introduction. With a robust time-domain code for computing waves from nearly arbitrary physical worldlines and with a robust frequency-domain code capable of "mass producing" radiation reaction data for generic Kerr black hole orbits this problem should boil down to simple a matter of available CPU resources. Once we are in this state, we hope to produce waveforms efficiently enough that they can be used by workers looking at problems in LISA data analysis and waveform measurement (e.g., the "Mock LISA Data Challenge" [38, 39, 40, 41]). These 
waveforms are likely to be useful for other astrophysical problems, such as computing radiation recoil from both the slow inspiral and the dynamic plunge. Computing this effect in the extreme mass ratio limit may serve as a precision check on recent work looking at this problem in full numerical relativity [42, 43, 44, 45, 46].

\section{Acknowledgments}

We thank Lior M. Burko for very helpful discussions on various aspects of this work, particularly for suggesting the orbital-frequency dependence used in Eq. (5.1), and other discussions regarding the the impact of finite extraction radius on $\dot{E}$. We likewise thank Eric Poisson for helpful discussion regarding finite extraction radius. Many of the numerical simulations presented here were performed at the San Diego Supercomputing Center. GK acknowledges research support from the University of Massachusetts and Glaser Trust of New York, as well as supercomputing support from the TeraGrid (grant number TG-PHY060047T). PAS and SAH are supported by NASA Grant No. NNG05G105G; SAH is in addition supported by NSF Grant No. PHY-0449884, and gratefully acknowledges the support of the MIT Class of 1956 Career Development fund.
[1] C. Hopman, "Astrophysics of extreme mass ratio inspiral sources", in Laser Interferometer Space Antenna: 6th International LISA Symposium, AIP Conf. Proc. 873, 241 (2006); astro-ph/0608460

[2] See http://lisa.nasa.gov for an overview and description of the LISA mission.

[3] A. Pound, E. Poisson, and B. G. Nickel, Phys. Rev. D 71, 104003 (2005).

[4] J. R. Gair, L. Barack, T. Creighton, C. Cutler, S. L. Larson, E. S. Phinney, and M. Vallisneri, Class. Quantum Grav. 21, S1595 (2004).

[5] S. Drasco, E. E. Flanagan, and S. A. Hughes, Class. Quantum Grav. 22, S801 (2005).

[6] M. Favata, unpublished Ph. D. thesis, Cornell University (2006).

[7] M. Favata, in preparation. This paper expands on Chapter 4 of Ref. [6].

[8] E. E. Flanagan, and T. Hinderer, in preparation. This paper uses a "two timescale" analysis to systematically examine the validity of the adiabatic approach to inspiral in Kerr spacetimes; it finds and corrects an error in the conceptual foundation of Appendix A of Ref. [5], but shows that the numerical impact of this error does not change that reference's conclusion.

[9] S. A. Hughes, S. Drasco, E. E. Flanagan, and J. Franklin, Phys. Rev. Lett. 94, 221101 (2005).

[10] C. W. Misner, K. S. Thorne, and J. A. Wheeler, Gravitation (Freeman, San Francisco, 1973).

[11] S. Babak, H. Fang, J. R. Gair, K. Glampedakis, and S. A. Hughes, Phys. Rev. D 75, 024005 (2007).

[12] W. Schmidt, Class. Quantum Grav. 19, 2743 (2002).

[13] S. Drasco and S. A. Hughes, Phys. Rev. D 69, 044015 (2004).

[14] S. Drasco and S. A. Hughes, Phys. Rev. D 73, 024027 (2006).

[15] N. Sago, T. Tanaka, W. Hikida, and H. Nakano, Prog. Theor. Phys. 114, 509 (2005).

[16] N. Sago, T. Tanaka, W. Hikida, K. Ganz, and H. Nakano, Prog. Theor. Phys. 115, 873 (2006).

[17] R. Lopez-Aleman, G. Khanna, and J. Pullin, Class. Quantum Grav. 20, 3259 (2003).

[18] G. Khanna, Phys. Rev. D 69, 024016 (2004).

[19] L. M. Burko and G. Khanna, Europhys. Lett. 78, 60005 (2007).

[20] C. Sopuerto and P. Laguna, Phys. Rev. D 73, 044028
(2006).

[21] C. Sopuerta, "Time Domain Simulations of EMRIs using Finite Element Methods", in Laser Interferometer Space Antenna: 6th International LISA Symposium, AIP Conf. Proc. 873, 274 (2006); gr-qc/0609240.

[22] S. Teukolsky, Astrophys. J. 185, 635 (1973).

[23] A. K. Engquist, A. K. Tornberg, and R. Tsai, J. Comput. Phys. 207, 28 (2005).

[24] A. K. Tornberg and B. Engquist, J. Comput. Phys. 200, 462 (2004)

[25] W. Krivan, P. Laguna, P. Papadopoulos, and N. Andersson, Phys. Rev. D 56, 3395 (1997).

[26] K. D. Kokkotas, Class. Quantum Grav. 8, 2217 (1991).

[27] S. A. Hughes, Phys. Rev. D 61, 084004 (2000).

[28] M. Davis, R. Ruffini, W. H. Press and R. H. Price, Phys. Rev. Lett. 27, 1466 (1971).

[29] S. A. Teukolsky, Phys. Rev. Lett. 29, 16 (1972).

[30] E. Pazos-Avalos, C. O. Lousto, Phys. Rev. D, 72084022 (2005).

[31] P. A. Sundararajan, G. Khanna, S. Drasco and S. A. Hughes, in preparation.

[32] G. Khanna, R. Gleiser, R. Price and J. Pullin, New J. Phys., 2, 3.1 (2000) (http://www.njp.org).

[33] M. Campanelli and C. O. Lousto, Phys. Rev. D, 59, 124022 (1999).

[34] L. M. Burko, private communication.

[35] L. M. Burko, S. A. Hughes, and P. A. Sundararajan, in preparation.

[36] E. T. Newman and T. W. J. Unti, J. Math. Phys. 3, 891 (1962).

[37] A. Ori and K. S. Thorne, Phys. Rev. D 62, 124022 (2000).

[38] K. A. Arnaud, S. Babak, J. G. Baker, M. J. Benacquista, N. J. Cornish, C. Cutler, S. L. Larson, B. S. Sathyaprakash, M. Vallisneri, A. Vecchio, and J.-Y. Vinet, "An Overview of the Mock LISA Data Challenges", in Laser Interferometer Space Antenna: 6th International LISA Symposium, AIP Conf. Proc. 873, 619 (2006); gr-qc/0609105.

[39] K. A. Arnaud, S. Babak, J. G. Baker, M. J. Benacquista, N. J. Cornish, C. Cutler, S. L. Larson, B. S. Sathyaprakash, M. Vallisneri, A. Vecchio, and J.Y. Vinet, "A How-To for the Mock LISA Data Challenges", in Laser Interferometer Space Antenna: 6th International LISA Symposium, AIP Conf. Proc. 873, 625 (2006); gr-qc/0609106. 
[40] N. J. Cornish and E. K. Porter, "Searching for Massive Black Hole Binaries in the first Mock LISA Data Challenge", to appear in the proceedings of the 11th Gravitational-wave Data Analysis Workshop; gr-qc/0701167.

[41] K. A. Arnaud, S. Babak, J. G. Baker, M. J. Benacquista, N. J. Cornish, C. Cutler, S. L. Larson, B. S. Sathyaprakash, M. Vallisneri, A. Vecchio, and J.-Y. Vinet, "An overview of the second round of the Mock LISA Data Challenges", to appear in the proceedings of the 11th Gravitational-wave Data Analysis Workshop; gr-qc/0701170
[42] J. G. Baker, J. Centrella, D.-I. Choi, M. Koppitz, J. R. van Meter, and M. C. Miller, Astrophys. J. 653, L93 (2006).

[43] J. A. Gonzalez, U. Sperhake, B. Brügmann, M. Hannam, S. Husa, gr-qc/0610154.

[44] F. Herrmann, I. Hinder, D. Shoemaker, P. Laguna, and R. A. Matzner, gr-qc/0701143

[45] M. Koppitz, D. Pollney, C. Reisswig, L. Rezzolla, J. Thornburg, P. Diener, and E. Schnetter, gr-qc/0701163

[46] M. Campanelli, C. O. Lousto, Y. Zlochower, and D. Merritt, gr-qc/0701164 